\title{
Optical manipulation of nanoparticles: a review
}

\author{
Maria Dienerowitz, Michael Mazilu, and Kishan Dholakia
}

\author{
SUPA, School of Physics and Astronomy, University of St Andrews, St Andrews, Fife, \\ KY169SS, United Kingdom \\ md60@st-andrews.ac.uk
}

\begin{abstract}
Optical trapping is an established field for movement of micron-size objects and cells. However, trapping of metal nanoparticles, nanowires, nanorods and molecules has received little attention. Nanoparticles are more challenging to optically trap and they offer ample new phenomena to explore, for example the plasmon resonance. Resonance and size effects have an impact upon trapping forces that causes nanoparticle trapping to differ from micromanipulation of larger micron-sized objects. There are numerous theoretical approaches to calculate optical forces exerted on trapped nanoparticles. Their combination and comparison gives the reader deeper understanding of the physical processes in an optical trap. A close look into the key experiments to date demonstrates the feasibility of trapping and provides a grasp of the enormous possibilities that remain to be explored. When constructing a single-beam optical trap, particular emphasis has to be placed on the choice of imaging for the trapping and confinement of nanoparticles.
\end{abstract}

Keywords: optical trapping and manipulation, optical tweezers, nanoparticles, particle plasmons, Rayleigh and Mie scattering, optical Lorentz force.

\section{INTRODUCTION}

The last thirty years have seen a revolution in our understanding of the light-matter interaction. The advent of the laser sparked numerous major research areas and one of these has been the trapping and manipulation of matter by light. Indeed the advances seen at the atomic scale level have led to the formation of quantum degenerate gases, now established as a standard experimental tool in many research laboratories. In tandem with the experimental advances, we have seen a deeper understanding of the complexity of the interaction of light and matter that has led to ultra-cold gases breaking the so-termed Doppler limit and the ever-growing list of elements which may now laser cooled to sub milli-Kelvin temperatures. These advances have been recognised with award of two Nobel prizes, in 1997 and 2001.

The impact of the light matter interaction has also occurred on scales larger than that of the atomic regime: the pioneering work of Ashkin led to the development of optical trapping of microscopic particles including biological specimens such as cells. Optical trapping at this scale has led a major revolution in single-molecule biophysics as they offer well calibrated force transducers that may measure forces right down to femto-Newtons. Using trapped beads as anchors to indirectly move macromolecules, researchers have made major strides into our understanding of molecular systems such as the actin-myosin system, kinesin motion on microtubules and DNA as well as opening up new studies of non-equilibrium statistical physics. Whilst optical trapping at the microscopic scale is well documented with various reviews [1-3] little attention has been paid to the trapping and manipulation of nanometric sized particles which falls largely in the size region between cells and that of atomic ensembles. In particular the trapping of metallic particles at the nanometre scale has gained momentum as well as the direct manipulation of biological macromolecules.

Nanoparticles span the entire size range from some tens of nanometres (the Rayleigh regime) up to a couple of hundred nanometres. Optical trapping of nanometric objects is still considered to be more challenging than trapping micron-sized objects. The fundamental limitation is the 
amount of force one can possibly exert on a particle by light on objects of such dimensions. The magnitude of the force depends upon the relative refractive index and size of the particle and reduces with decreasing particle volume and lower refractive index difference compared to the host medium. Despite this, it is possible to manipulate and control atoms by exploiting the enhancement of forces when operating near an internal atomic resonance.

Nanoparticle manipulation is a highly topical and powerful area for future studies with optical traps and is the focus of this review article. First we present the theoretical aspects of nanoparticle trapping. In the second chapter we describe the major experiments performed to date in this area. In the last part we include advice upon the construction of an optical trap with particular emphasis upon the trapping and visualisation of nanoparticles.

\section{THEORY}

In general, the change of direction or absorption of a light beam by a body gives rise to a momentum transfer from the light beam to the body. Within the photon description of light, each individual photon travels according to the geometrical optics ray picture and transfers all or part of its linear momentum to the body via an elastic or inelastic collision $[4,5]$. This description can be further enhanced by using a mixed geometrical optics and paraxial approximation approach to the light beam [6] and thus enable the treatment of focused laser beams. This needs high order corrections to account for beam focussing. Unfortunately, these methods are not directly applicable in the case of nanoparticles because of their restricted size compared to the wavelength. In this size regime, the local electromagnetic field and its phase play a fundamental role in the theoretical modelling of the optical momentum transfer. Further, depending on their size, the optical properties of nanoparticles differ from the bulk materials. In the case of metals, for example, this dependence becomes important when the nanoparticle size becomes comparable to the mean free path of the conduction electrons $[7,8]$.

In this section, we start by reviewing the size dependent corrections of the optical properties of metallic nanoparticles. Then, we present two methods to calculate the optical forces acting on nanoparticles: a general and a more specific approach. The general method is applicable to any size and shaped particles and we review four different but equivalent approaches, namely: the Maxwell stress tensor, two distinct formulations of Lorentz forces and optical surface forces. The more specific methods considered here take the reduced size of the nanoparticles into account and consist of the generalised Lorenz-Mie scattering, dipole approximation, and the sum of the optical gradient and scattering forces.

We have used standard notations for the basic quantities and parameters: $\mathbf{E}$ and $\mathbf{H}$ are the electric and magnetic fields. The macroscopic electromagnetic properties of a medium are defined by its electronic scattering time $\tau$, permittivity $\epsilon(\omega)$, permeability $\mu(\omega)$ and electric conductivity $\sigma(\omega)$. The latter are all functions of $\omega$, the angular frequency of light. The subscript $n$ and $h$ stand for the medium properties of the nanoparticle and host solution while the subscripts $r$ and 0 stand for the relative and vacuum constants respectively. Bold letters refer to vector quantities while their non bold version with the subscripts $i, j$ and $k$ stand for their three Cartesian coordinates each subscript varying from 1 to 3.

\subsection{Optical properties}

The successful modelling of the optical forces acting on nanoparticles relies upon the knowledge of their interaction with the electromagnetic field. Within classical electromagnetism, this is governed by Maxwell's equations and depends on the shape of the nanoparticle, its dielectric function $\epsilon_{n}(\omega)$ and the incident field. Here, we discuss the change of index of refraction $n_{n}^{2}=$ $\epsilon_{n}(\omega)$ due to the reduced size of the nanoparticle assuming a monochromatic field of the form $\exp (i \omega t)$ where $\omega$ is the fields optical frequency. Note that with this sign convention, absorptive nanoparticles have a negative imaginary index of refraction. 
The index of refraction in a material depends on its electronic properties and the ability of the electrons to change their configuration under the influence of the electromagnetic field. In general, the index of refraction is an effective medium concept in which the microscopic polarisability is homogenised over the whole particle replacing the local polarisation by an effective refractive index. The homogenisation method assumes that the individual molecules or atoms of the material are all in the same average environment equivalent to an infinitely extended bulk material. This assumption is perfectly valid for dielectric nanoparticles where the local polarisability originates from bound electrons, but it is not the case for metallic nanoparticles where the close proximity of the particle surface changes the electronic behaviour of the conduction electrons. Indeed, the mean free path of conduction electrons is given by $\tau_{b u l k} v_{f}$, where $v_{f}$ is the bulk Fermi velocity and $\tau_{b u l k}$ the bulk electronic scattering time (mean time between successive collisions). In bulk gold, at room temperature, this mean free path is about $42 \mathrm{~nm}$ [9]. For metallic nanoparticles with a diameter below this dimension, we need to consider the reduced scattering time for the conduction electrons, which is given by:

$$
\frac{1}{\tau_{n}}=\frac{1}{\tau_{b u l k}}+\frac{v_{f}}{a}
$$

where $a$ is the radius of the nanoparticles and $\tau_{n}$ the corrected particle electronic scattering time. The size corrected mean free path is given by $\tau_{n} v_{f}$. This change of the mean free path modifies the overall conductivity of the nanoparticle inducing a change of its dielectric function,

$$
\epsilon_{n}(\omega)=\epsilon_{\text {bound }}(\omega)+\epsilon_{\text {cond }}(\omega),
$$

where the dielectric functions $\epsilon_{\text {bound }}(\omega)$ and $\epsilon_{\text {cond }}(\omega)$ represent the part of the dielectric function originating from the bound and conduction electrons respectively [10]. The bound component is not affected by the size of the nanoparticle while the conduction part is affected. It is possible to use the experimentally measured bulk dielectric function $\epsilon_{\text {bulk }}(\omega)$ and to correct it by taking into account the mean free path change within the Drude model,

$$
\epsilon_{n}(\omega)=\left[\epsilon_{\text {bulk }}(\omega)-\frac{4 \pi \sigma_{\text {bulk }}}{\omega\left(1+i \omega \tau_{b u l k}\right)}\right]+\frac{4 \pi \sigma_{n}}{\omega\left(1+i \omega \tau_{n}\right)},
$$

where $\sigma_{\text {bulk }}=\omega_{n} 2 \tau_{\text {bulk }} / 4 \pi, \sigma_{n}=\sigma_{\text {bulk }} \tau_{n} / \tau_{\text {bulk }}$ and $\omega_{p}$ are the bulk conductivity, particle conductivity and the plasma frequency respectively. A more elaborate description including a quantum-mechanical treatment of the size dependence of the optical properties of metallic nanoparticles can be found in [11] while the size dependent contribution from bound electrons is taken into account in [12].

Table 1. Optical properties for gold nanoparticles $[13,14]$ at a wavelength of $500 \mathrm{~nm}$ and with a Fermi velocity $v_{f}=1.41 \cdot 10^{6} \mathrm{~m} / \mathrm{s}[7,8]$.

\begin{tabular}{|c|c|c|c|c|}
\hline & $\sigma_{n}\left(10^{17} \mathrm{~s}^{-1}\right)$ & $\tau_{n}\left(10^{-15} \mathrm{~s}\right)$ & $\epsilon_{n}$ & $n_{n}$ \\
\hline Bulk gold & 3.70 & 24.7 & $-2.68-3.09 \mathrm{i}$ & $0.84-1.84 \mathrm{i}$ \\
$100 \mathrm{~nm}$ gold & 2.18 & 14.6 & $-2.68-3.19 \mathrm{i}$ & $0.86-1.85 \mathrm{i}$ \\
$10 \mathrm{~nm}$ gold & 0.46 & 3.10 & $-2.59-4.08 \mathrm{i}$ & $1.06-1.92 \mathrm{i}$ \\
\hline
\end{tabular}

In addition to the dielectric function depending on the size of one individual nanoparticle, we also need to consider their collaborative effect on the propagation of the incident beam when it traverses regions of dispersed nanoparticles. In this case, it is possible to use a generalised 
Maxwell-Garnett homogenisation theory [15] that determines the effective dielectric function of the suspension depending on the concentration and shape of the nanoparticles. For spherical particles in a host with $\epsilon_{h}(\omega)$, we have:

$$
\frac{\epsilon_{e f f}(\omega)-\epsilon_{h}(\omega)}{\epsilon_{e f f}(\omega)+2 \epsilon_{h}(\omega)}=(1-x) \frac{\epsilon_{n}(\omega)-\epsilon_{h}(\omega)}{\epsilon_{n}(\omega)+2 \epsilon_{h}(\omega)},
$$

where $x$ is the volume fraction of the host and $\epsilon_{\text {eff }}(\omega)$ denotes the total effective dielectric function of the solution.

\subsection{Momentum conservation}

The fundamental principle involved in optical forces is the conservation of the electromagnetic momentum-energy when light scatters from a particle. Incident light that is scattered or absorbed by a particle is subject to a change in momentum which is transferred to the mechanical momentum of the particle. The optical force acting on a nanoparticle can thus be deduced from integrating the change of momentum of the electromagnetic field as a whole. In the following, we consider a monochromatic light field of optical frequency $\omega$ and the particles to be quasistationary such that Doppler shift effects are negligible. In the linear case, the monochromatic assumption can be generalised to deal with broad spectra by summing all the optical forces resulting from the individual spectral components. Indeed, in the stationary assumption, the total optical force is independent of the relative phase/chirp of the spectrum of the incident light field. Finally, we also note that non-stationary particles are dealt with by considering both momentum conservation and energy conservation.

For electromagnetic waves, the momentum density in a medium with relative permittivity $\epsilon_{h}$ and permeability $\mu_{h}$ is defined by the Maxwell stress tensor, $T_{i j}$,

$$
T_{i j}=\epsilon_{h} \epsilon_{0} E_{i} E_{j}^{*}+\mu_{h} \mu_{0} H_{i} H_{j}^{*}-\frac{1}{2} \delta_{i j}\left(\epsilon_{h} \epsilon_{0} E_{k} E_{k}^{*}+\mu_{h} \mu_{0} H_{k} H_{k}^{*}\right),
$$

where we sum over repeating indices and the superscript star corresponds to the complex conjugate. This stress tensor gives the flux of momentum across an area and is the starting point in all field based optical force calculations. The stress tensor itself is not uniquely defined. Its precise form depends on the conceptual split of the total electromagnetic momentum into two parts: the momentum carried by the electromagnetic wave and the momentum carried by the induced polarisation in the medium the wave is propagating through. Fortunately, this split does not affect the total optical force acting on the particle [16,17]. This total optical force (see curve A in Fig. 1 and Fig. 2) is given by integrating the momentum flux flow over the surface of the particle:

$$
\begin{aligned}
<F_{i}> & =\frac{1}{2} \Re\left(\int_{S} T_{i j} n_{j} d s\right) \\
& =\int_{V}<f_{i}>d v=\int_{V} \frac{1}{2} \Re\left(\partial_{j} T_{i j}\right) d v,
\end{aligned}
$$

where $V$ is the volume of the particle, $S$ its surface and $n_{j}$ the vector components of the normal pointing outwards from this surface. The brackets $\langle\ldots\rangle$ correspond to the time average over an optical cycle $T=2 \pi / \omega$ and $\Re$ to the real part of a complex number. Here, we also introduced the optical force density $f_{i}$, visualising a volumetric density of force across the particle. The described Maxwell stress tensor method is a versatile tool and applied in a variety 
a)

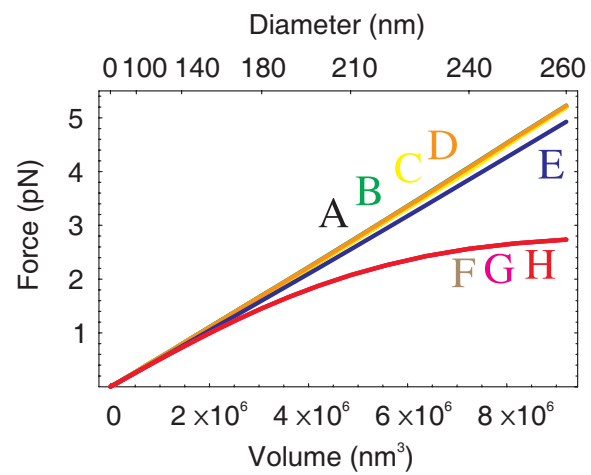

b)

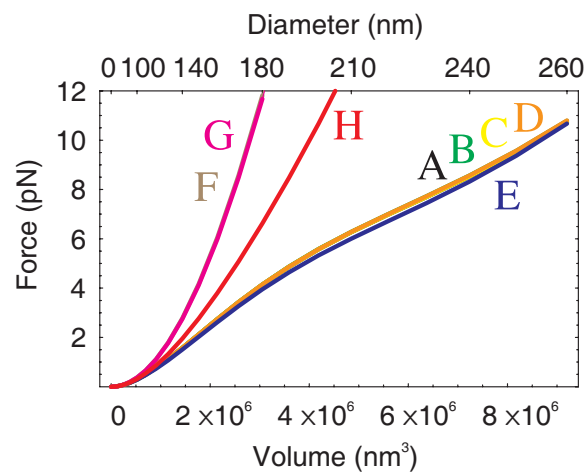

Fig. 1. Optical forces acting on a dielectric nanoparticle $\left(n_{n}=1.59\right)$ in water as a function of its volume (lower x-axis) and diameter (upper x-axis). The nanoparticle is in the focal plane of a linearly polarised Gaussian beam (waist $w_{0}=1 \mu \mathrm{m}$, wavelength $\lambda=500 \mathrm{~nm}$ ) laterally offset by $300 \mathrm{~nm}$. The transversal (a) and longitudinal (b) optical forces acting are calculated for the different models: A Maxwell's stress tensor Eq.(5); B Surface gradient forces Eq.(11); C Lorentz force Eq.(8); D Lorentz force Eq.(9); E Lorenz-Mie forces; F Gradient and scattering forces Eq.(14); G Dipole approximation forces Eq.(17); H Gradient and scattering forces using Lorenz-Mie scattering and absorption coefficients.

of different scenarios, ranging from nanoparticles in evanescent fields $[18,19]$ and arrays of nanoparticles [20,21], just to mention a few examples.

An alternative approach is to calculate the total optical force using the Lorentz force. This leads to a different optical force density distribution which, when integrated across the volume of the particle, gives the same total force as the force density in Eq.(6). Indeed, the electric field induces a local polarisation which gives rise to a current on which the electromagnetic field acts through the Lorentz force. This Lorentz optical force density reads [22]:

$$
<\mathbf{f}>=\frac{1}{2} \Re\left[(\mathbf{P} \cdot \nabla) \mathbf{E}^{*}+\partial_{t} \mathbf{P} \times \mu_{0} \mathbf{H}^{*}\right],
$$

where $\mathbf{P}=\left(\epsilon_{n}-\epsilon_{h}\right) \mathbf{E}$ is the relative polarisation in the particle with respect to the surrounding medium (see curve $\mathrm{C}$ in Fig. 1 and Fig. 2). Alternatively, the Lorentz force density can be written as [23]:

$$
<\mathbf{f}>=\frac{1}{2} \Re\left[-(\nabla \cdot \mathbf{P}) \mathbf{E}^{*}+\partial_{t} \mathbf{P} \times \mu_{0} \mathbf{H}^{*}\right]
$$

(see curve D in Fig. 1 and Fig. 2). This force density is again different from the two previous ones (Eq. (6) and (8)) but gives rise to the same total optical force. Equations (8) and (9) consider only non magnetic host and nanoparticles $\left(\mu_{h}=1\right)$. Including a magnetic dipole into the force calculation leads to the complete Lorentz force density [24]. The additional forces become important when considering meta-material nanoparticles and are expressed as:

$$
<\mathbf{f}>=\frac{1}{2} \Re\left(\rho_{e} \mathbf{E}^{*}+\rho_{m} \mathbf{H}^{*}+\mathbf{j}_{\mathbf{e}} \times \mu_{\mathbf{0}} \mathbf{H}^{*}+\mathbf{j}_{\mathbf{m}} \times \epsilon_{\mathbf{0}} \mathbf{E}^{*}\right),
$$

where $\rho_{e}=-\nabla \cdot \mathbf{P}_{e}, \mathbf{j}_{\mathbf{e}}=\partial_{\mathbf{t}} \mathbf{P}_{\mathbf{e}}, \rho_{m}=-\nabla \cdot \mathbf{P}_{m}$ and $\mathbf{j}_{\mathbf{m}}=\partial_{\mathbf{t}} \mathbf{P}_{\mathbf{m}}$ correspond to the electric and magnetic densities and currents. These in turn, depend on the electric and magnetic polarisation $\mathbf{P}_{e}=\left(\epsilon_{n}-\epsilon_{h}\right) \mathbf{E}$ and $\mathbf{P}_{m}=\left(\mu_{n}-\mu_{h}\right) \mathbf{H}$. 
It is also possible to express the optical force as a surface force which acts on the interface between different media. This force density is defined by further simplifying Eq.(5), which leads to:

$$
\begin{aligned}
<\mathbf{f}>= & -\frac{\epsilon_{0}}{4}\left(\mathbf{E} \cdot \mathbf{E}^{*}\right) \nabla\left(\epsilon_{r}^{\prime}\right)-\frac{\mu_{0}}{4}\left(\mathbf{H} \cdot \mathbf{H}^{*}\right) \nabla\left(\mu_{r}^{\prime}\right) \\
& +\frac{\epsilon_{r}^{\prime \prime} \epsilon_{0}}{2} \Im\left[\left(\mathbf{E}^{*} \cdot \nabla\right) \mathbf{E}\right]+\frac{\mu_{\mathbf{r}}^{\prime \prime} \mu_{\mathbf{0}}}{\mathbf{2}} \Im\left[\left(\mathbf{H}^{*} \cdot \nabla\right) \mathbf{H}\right] \\
& -\frac{\omega}{2} \Im\left(\epsilon_{r} \mu_{r}\right) \Re\left(\mathbf{E}^{*} \times \mathbf{H}\right),
\end{aligned}
$$

which generalises $[25,26]$ to include absorptive and magnetic media (see curve B in Fig. 1 and Fig. 2). For convenience, we have split the relative local permittivity and permeability $\epsilon_{r}=\epsilon_{r}^{\prime}+i \epsilon_{r}^{\prime \prime}$ and $\mu_{r}=\mu_{r}^{\prime}+i \mu_{r}^{\prime \prime}$ into real and imaginary parts. Contrary to the Lorentz force density discussed earlier, this force density places the force on the surface formed by the discontinuity between the particle and the host material and implies bulk forces only in presence of absorption by the particle.

Regardless of the form used, if applied correctly, all these notations give the same total optical force and they all have one thing in common: in order to evaluate these expressions, we need the knowledge of the field interacting with the particle i.e. we need to solve Maxwell's equations, taking into account the incident field and its scattering by the particle. There are a variety of methods to solve this problem and it is the restricted size of the nanoparticle playing a role in the choice of the method used. In the following, we consider three different approaches that take into account the reduced size of the nanoparticle. These are the generalised LorenzMie scattering, the dipole approximation, and the sum of the optical gradient and scattering forces.

\subsection{Rayleigh and Lorenz-Mie scattering}

In general, for particles smaller than $\lambda / 20$, we can calculate the optical forces using the Rayleigh scattering coefficients [27]. This approximation is also called the quasi static theory [28] as it assumes the field to be constant across the particle i.e. it is valid for particles much smaller than the wavelength. For these point-like dipole particles it is possible to estimate their total microscopic polarisation as proportional to the incident electric field. The induced polarisation of these particles is given by $\mathbf{p}=\epsilon_{\mathbf{0}} \epsilon_{\mathbf{h}} \alpha \mathbf{E}$, where $\alpha$ is the polarisability. The induced polarisation varies together with the incident electric field implying an oscillating dipole that is associated with a current $\mathbf{j}=\partial_{\mathbf{t}} \mathbf{p}$. This current, in conjunction with the optical Lorentz force (Eq. (8) for example), gives the total optical force acting on the nanoparticle $[29,30]$

$$
\left.<\mathbf{f}>=\frac{\epsilon_{0} \epsilon_{h}}{2} \Re\left[\alpha(\mathbf{E} \cdot \nabla) \mathbf{E}^{*}+\alpha \partial_{t} \mathbf{E} \times \mu_{0} \mathbf{H}^{*}\right)\right] .
$$

Conceptually, this force can be split into the sum of two parts, namely the gradient force and the scattering force. This approach to calculating optical forces is very useful as it gives a direct and quick method for predicting the optical forces acting on nanoparticles by observing the incident field (see the experimental work in Sec. 3.2.1 and Sec. 4.3 for examples). The gradient force causes the nanoparticles to be attracted by high intensity parts of the field, such as the focal spot, and depends on the gradient of intensity of the incident beam:

$$
<\mathbf{F}_{\text {grad }}>=\frac{\epsilon_{0} \epsilon_{h}}{4} \Re(\alpha) \nabla\left(\mathbf{E} \cdot \mathbf{E}^{*}\right),
$$


a)

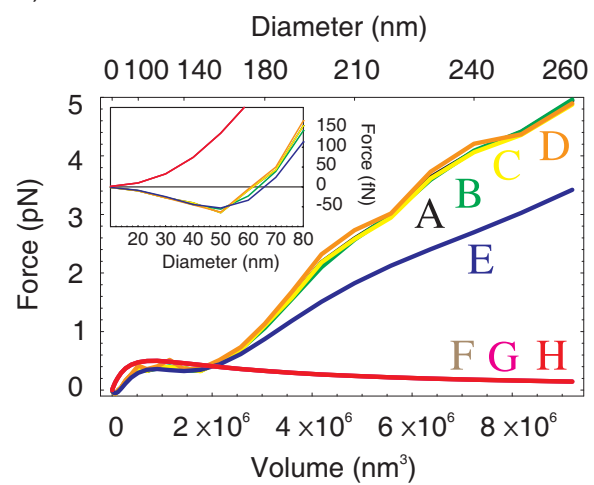

b)

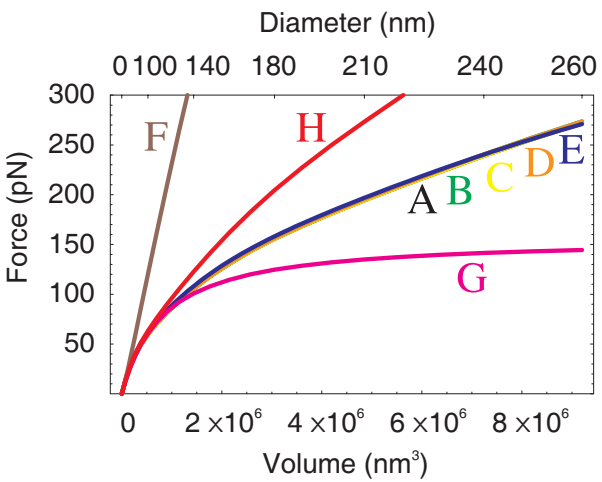

Fig. 2. Optical forces acting on a gold nanoparticle $\left(n_{n}=0.86-1.85 i\right)$ in water as a function of its volume (lower $\mathrm{x}$-axis) and diameter (upper x-axis). The transversal (a) and longitudinal (b) optical forces acting are calculated for the different models: A Maxwell's stress tensor Eq.(5); B Surface gradient forces Eq.(11); C Lorentz force Eq.(8); D Lorentz force Eq.(9); E Lorenz-Mie forces; F Gradient and scattering forces Eq.(14); G Dipole approximation forces Eq.(17); H Gradient and scattering forces using Lorenz-Mie scattering and absorption coefficients. The inset shows small the particle limit.

where we used the vector calculus identity $\nabla\left(\mathbf{E} \cdot \mathbf{E}^{*}\right)=2 \Re\left[(\mathbf{E} \cdot \nabla) \mathbf{E}^{*}+\mathbf{E} \times\left(\nabla \times \mathbf{E}^{*}\right)\right]$ and Maxwell's equation $\nabla \times \mathbf{E}=-\mu_{\mathbf{0}} \partial_{\mathbf{t}} \mathbf{H}$. The nature of the gradient force depends on the sign of the real part of the polarisability. The gradient force corresponds to the zeroth order approximation of the total optical force. Indeed, this force depends only on the incident optical intensity and does not directly involve the amount of optical interaction with the incident field.

The scattering force corresponds to a first order approximation term of the optical force originating in the momentum loss or transfer from the incident light to the particle. The momentum transfer/loss is directly linked to the amount of scattering and absorption of the particle, taking its optical interaction with the electromagnetic field into account. This force is plotted in Figs. 1 and Fig. 2 (see curve $\mathrm{F}$ and $\mathrm{H}$ ) and is given by:

$$
<\mathbf{F}_{\text {scat }}>+<\mathbf{F}_{a b s}>=\frac{n_{h}}{c} C_{\text {scat }}<\mathbf{S}>+\frac{n_{h}}{c} C_{a b s}<\mathbf{S}>,
$$

where $\left\langle\mathbf{S}>=1 / 2 \Re\left(\mathbf{E} \times \mathbf{H}^{*}\right)\right.$ is the optical cycle averaged Poynting vector and where $C_{\text {scat }}=n_{h}^{4} k_{0}^{4} /(6 \pi)|\alpha|^{2}$ and $C_{a b s}=-n_{h} k_{0} / \epsilon_{0} \Im\left(\alpha_{0}\right)$ are the scattering and absorption cross sections within the Rayleigh approximation $\left(k_{0}\right.$ is the vacuum wavevector and $\Im$ denotes the imaginary part of a complex number). The use of these cross sections is valid for incident fields that vary slowly in amplitude compared to the size of the nanoparticle.

In order to evaluate the gradient and the scattering forces we need to determine the polarisability of the particle. This is obtained through the Clausius-Mossotti homogenisation procedure with

$$
\begin{aligned}
\alpha_{0} & =4 \pi a^{3} \frac{\epsilon_{n}-\epsilon_{h}}{\epsilon_{n}+2 \epsilon_{h}}, \\
\alpha & =\alpha_{0} /\left[1+i n_{h}^{3} k_{0}^{3} \alpha_{0} /(6 \pi)\right],
\end{aligned}
$$

where $a$ corresponds to the radius of the nanoparticle. Here, the standard polarisability $\alpha_{0}$ is corrected to include the radiative reaction of the nanoparticle [31]. The radiative reaction correction takes into account the amount of scattering by the nanoparticle corresponding effectively to the attenuation seen by the incident filed. The correction leads to a complex polarisability, 
dephasing the oscillation of the dipole with respect to the incident field, effectively absorbing the incident field. Additionally, it is possible to include into the microscopic polarisability the internal structure of the nanoparticle such as nanoshell fabricated by coating nanoparticles [28].

The slowly varying amplitude restriction can be relaxed through the use of a more general Rayleigh force formula which is deduced from the second form of the Lorentz force in Eq.(9). Again, considering a nanoparticle with a microscopic polarisability $\alpha=\alpha^{\prime}+i \alpha^{\prime \prime}$, we have [32]:

$$
\begin{aligned}
<F_{i}>_{\text {Rayleigh }} & =\frac{\epsilon_{0} \epsilon_{h}}{2} \Re\left(\alpha E_{j} \partial_{i} E_{j}^{*}\right) \\
& =\frac{\epsilon_{0} \epsilon_{h} \alpha^{\prime}}{4} \partial_{i}\left(E_{j} E_{j}^{*}\right)+\frac{\epsilon_{0} \epsilon_{h} \alpha^{\prime \prime}}{2} \Im\left(E_{j}^{*} \partial_{i} E_{j}\right),
\end{aligned}
$$

where the total Rayleigh force is split into a gradient and a scattering force [33] (see curve G in Fig. 1 and Fig. 2). In this instance, the radiative correction becomes important as, without it, dielectric particles would have a purely real polarisability and thus would not be subject to scattering forces.

As the size of the nanoparticle increases, its optical behaviour becomes more complex and can no longer be described by a simple dipole model but needs to be generalised to include multipole effects. This is achieved within the Lorenz-Mie scattering theory which decomposes an incident plane wave or, more generally, an arbitrary beam into vector spherical harmonics. Imposing the continuity of the tangential fields at the spherical interfaces it is possible to define Mie coefficients similar to the transmission and reflection in multi-layered structures [34-36]. Knowing these Mie coefficients allows us to analytically integrate Maxwell's stress tensor around the particle and to determine the amount of momentum transferred to the particle in the Lorenz-Mie scattering process (see curve E in Fig. 1 and Fig. 2).

Within the generalised Lorenz-Mie scattering theory, the incident beam is assumed to be a correct solution to Maxwell's equations that is decomposed onto the incident spherical harmonic fields. Tightly focussed beams can be described using higher order corrections to Gaussian beams [37]. Decomposing these beams into the infinite series of spherical harmonics proves to be a challenge because of the slow convergence. Alternative procedures involve the description of the incident beam using the localised beam model [38,39], partial waves [40] or s-expansion methods [41]. The treatment of non-spherical particles is accomplished within the T-matrix method which is equivalent to Lorenz-Mie scattering for spherical particles [42-44].

In Fig. 1 and Fig. 2 we compare the theoretical calculated optical forces acting on nanoparticles as a function of their size. For all eight methods considered, we calculated the force along (longitudinal) and across (transversal) the direction of propagation of a linearly polarized Gaussian beam having a total power of $P_{0}=1 \mathrm{~W}$, a focal waist $w_{0}=1 \mu \mathrm{m}$ and a wavelength of $\lambda=500 \mathrm{~nm}$. The nanoparticle is positioned in the focal plane of the beam $300 \mathrm{~nm}$ from its axis along the polarization direction. We calculated the optical forces for a dielectric particle and for a metallic nanoparticle. To facilitate the observation of the linear link between the nanoparticle volume and optical force we chose to represent these forces as a function of the total particle volume. For convenience, we indicate the corresponding diameter of the nanoparticle on the top $\mathrm{X}$-axis.

We observe that all four methods based on Maxwell's stress tensor (see curves A-D in Fig. 1- 2) give the same optical forces within the numerical error. All these four methods are based on calculating the local electromagnetic fields and deriving the amount of momentum transfer to the nanoparticle from these fields. This is calculated either by considering the flux of momentum across the particles boundary or through the local Lorentz force density. It is thus not surprising that even though the four force field densities are different they all lead to the same total force. Lorenz-Mie based force calculation is also an exact approach. The difference 
between the forces calculated using Lorenz-Mie and the local field methods is due to the different description of the incident beam which is mostly visible for the transversal force in the case of the metallic nanoparticle. Indeed, due to its absorption, the metallic particle is sensitive to the precise phase profile of the Gaussian beam. The phase profile is the only difference between the two representations. We also observe that the approximate dipole based methods work quite well for very small particles except in the case of the transversal force calculation for metallic nanoparticles. Here, these methods fail to take into account the plasmonic resonance of the particle leading to the repulsive transversal force as calculated by the exact methods. This feature is not present in the case of dielectric nanoparticles.

Further, in Fig. 2b, we observe that the linear relationship between volume and longitudinal force beaks down in the case of metallic nanoparticles. For very small nanoparticles the linearity is preserved whereas the force acting on larger sized particles varies as the square of their diameter. This effect is linked to the skin depth of the metallic particles. If the size of the nanoparticle is smaller then its skin depth, the field interacts with the whole volume of the particle resulting in a linear dependence between force and volume. For nanoparticle sizes larger than the skin depth, the field interacts only with a thin outer layer of the nanoparticle. This layer increases with the surface of the particle, giving rise to a quadratic behaviour of the force as a function of the particles radius. Fig. $2 \mathrm{~b}$ shows the transition between these two regimes as a change of slope.

\section{EXPERIMENTS}

Optical trapping of nanometric objects is a rapidly growing field and its relevance is already stretching beyond physics towards biology and chemistry. In this chapter we highlight experimental achievements to date and point out remaining challenges. There are various kinds of nanoparticles: quantum dots, molecules, dielectric, semiconductor and metal nanoparticles all very exciting and interesting objects of study that would benefit from controlled manipulation. Nanoparticles are larger than atoms but smaller than the commonly tweezed micron-sized beads and cells. Most of them have resonances (excitons, plasmons, fluorophores, cavities) affecting their trapping properties. In order to address the different facets of these various types of nanoparticles, we divide this chapter into three sections: optical trapping of dielectric, metallic and molecular nanoparticles.

\subsection{Trapping dielectric nanoparticles}

The force exerted on a trapped object is fundamentally limited by size and relative refractive index (the ratio of the refractive index of the trapped object and the host medium). It decreases with the particle's polarisability $\alpha$ which is proportional to the volume of the particle. Thus this force is small for nanoparticles and even smaller for dielectrics because of the low index difference to the host medium. Consequently high powers are required to trap a dielectric nanoparticle with single-beam optical tweezers [45]. Alternative trapping geometries such as counter-propagating beams have proved to be more favourable than single-beam tweezers [46]. Extending one dimension of the dielectric nanoparticle to micron-size increases the volume of the trapped particle. This enhances the force that can be exerted on the trapped object such as carbon nanotubes or semiconductor nanowires [47,48].

So far, there have been very few experimental attempts to trap dielectric nanoparticles [45, 49]. Already in his pioneering optical tweezing experiment in 1986, Ashkin pointed out the challenges for trapping very small dielectric beads [45]. He derived a stability criterion depending upon the size, the refractive index of the particle and the beam waist of the applied laser. A very important constraint emerged in the experiment: the power needed to tweeze a particle may exceed the damage threshold of the particle itself. Ashkin demonstrated optical tweezing of differently sized dielectric spheres ranging from $10 \mu \mathrm{m}$ down to $25 \mathrm{~nm}$. The required laser 
a)

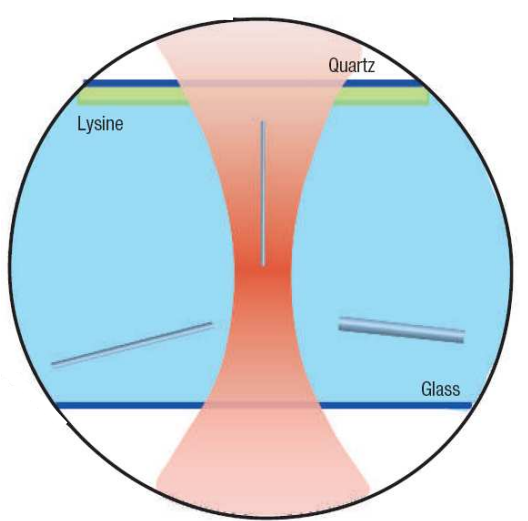

b)

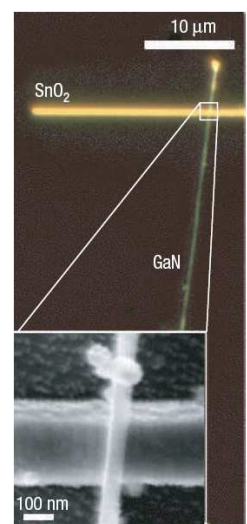

Fig. 3. a) The nanowires sink to the bottom of the sample chamber where they are picked up with optical tweezers, here pictured from the side. b) $\mathrm{GaN}$ nanowires and $\mathrm{SnO} 2$ nanoribbons optically laser-fused after they were manipulated in the correct place with optical tweezers. Pauzauski et al. imaged the nanowire structures with darkfield microscopy (see Sec. 4.4). Adapted from [48] and reprinted by permission from Macmillan Publishers Ltd: Nature Materials, copyright (2006).

power increased with decreasing particle diameter, being up to $1.4 \mathrm{~W}$ for $25 \mathrm{~nm}$ silica particles. The silica spheres were immersed in water which is advantageous as the damping decreases the Brownian motion and hence it is more likely or easier to trap them as the Brownian escape times are increased. In the experiment, Ashkin used a single focused beam as a trapping laser which was inserted into a high numerical aperture water immersion microscope objective. The spheres were observed indirectly via the scattered light of the particles. This method even supplied an approach to determine the size of the silica spheres from observing the scattering pattern and comparing it to a sphere's scattering pattern of known size.

In a counter-propagating geometry the axial scattering force components balance from opposing directions and the essential gradient force component dominates the trap site. As a result it is possible to trap very small dielectric spheres at very low powers. Zemanek et al. designed a specific counter-propagating geometry where the Gaussian standing wave creates nodes and antinodes resulting in a very strong axial gradient force that exceeds the axial gradient force of single-beam tweezers [46]. As a result, this geometry is not as sensitive to beam aberrations and also works at lower numerical aperture as the optical tweezers system. Zemanek et al. demonstrated trapping of $100 \mathrm{~nm}$ polystyrene spheres at trapping powers as low as $7 \mathrm{~mW}$ in a so-called Gaussian standing wave trap. The trap is formed by a focussed Gaussian beam passing the sample chamber and reflected on a coated coverslip.

A very interesting area is the optical trapping of objects having 2 dimensions in the nanometre range and the third dimension is micron-sized. This is how we may consider nanowires and carbon nanotubes. Several groups have been working on manipulating carbon nanotubes [47, 50,51]. In all cases bundles of carbon nanotubes are trapped and manipulated. Single-beam tweezers have been used but Plewa $e t$ al. showed the potential of holographic tweezers arranging carbon nanotubes in various shapes and even rotating entire bundles [50]. Several groups report enhanced diffusion of carbon nanotubes into the trap site, however trapping of single carbon nanotubes or trap stiffness measurements have not been reported to date. Enhanced diffusion is also observed in molecular trapping experiments; we will discuss this further in Sec. 3.3.

Semiconductor nanowires or nanorods have also been optically manipulated with singlebeam tweezers [48,52]. Once trapped, the automatic alignment of the nanorod in the trap along the axis of the beam can be used as an advantage to manipulate the entire nanorod (Fig. 3). 
Agarwal et al. created several single-beam traps by means of holographic beamshaping and placed these along one nanowire [53]. Advancing this multiple beam geometry into counterpropagating tweezers, created with an acousto-optic deflector (AOD), enabled van der Horst et al. to freely manipulate nanowires in three dimensions [52]. A line trap is another trapping geometry that has been suggested by $\mathrm{Yu}$ et al. [53]. They manipulated $\mathrm{CuO}$ nanorods and precisely put these in place to bridge two electrodes. Nanorods can also be fused once they have been put in the correct place with optical tweezers and build more complex three dimensional structures $[48,54]$. In this case by shortly increasing the power, the trapping beam is used to fuse the nanorods. Furthermore there have been theoretical suggestions about resonantly based trapping of semiconductor nanoparticles (nanometric in all three dimensions), exploiting the excitonic resonance [55]. However it was argued whether the assumed narrow linewidths of the excitonic resonance are realistic [56]. In this particular case, a narrow linewidth is required to achieve strong optical forces in order to stably trap semiconductor nanoparticles.

\subsection{Trapping metal nanoparticles}

Metal nanoparticles exhibit particle plasmons, a resonant excitation of free electrons in the metal, and thus are more complex than their dielectric counterparts. An electromagnetic wave at the resonance wavelength excites the particle plasmon in the nanoparticle. This resonance wavelength is situated in the visible for gold and silver nanoparticles. If the wavelength of a trapping laser coincides with the plasmon resonance wavelength, the trapping properties of the nanoparticle are significantly altered. Manipulating metal nanoparticles close to their particle plasmon resonance thus requires a sensible choice of the laser wavelength. Conversely, the particle properties far from resonance remain constant over a broad wavelength range and optical trapping of metal nanoparticles far from resonance resembles trapping high refractive index dielectric nanoparticles. We first review the pioneering works of trapping metal nanoparticles. These experiments have been conducted far from the nanoparticle's plasmon resonance, preferably using a trapping wavelength at $1064 \mathrm{~nm}$. In the second part, we discuss the newest developments in plasmon resonance based trapping. For a detailed discussion of the theoretical aspects of the specific force components we refer the reader to Sec. 2.3.

\subsubsection{Non-resonant optical trapping}

In the early years of optical trapping it was believed that tweezing of metal particles was impossible [57]. Since metallic objects are highly reflective it was concluded that they were pushed out of any high intensity region of a trapping laser. In 1992, Svoboda and Block [27] performed a key experiment showing that it is indeed possible to optically tweeze metal nanoparticles. By choosing a trapping laser in the infrared they conducted their experiment far from the particle plasmon resonance. At this wavelength, the $40 \mathrm{~nm}$ gold nanoparticles have a large refractive index relative to the host medium und therefore the gradient force is increased as compared to a dielectric particle of the same size. This increase in polarisability is more dominant than the increase in scattering and absorption cross sections (see Sec. 2.3). The authors used an interferometric position detection; a precise method that relies on the interference of two trapping beams acting as one optical trap. The interference pattern is imaged on a photodetector that translates the optical signal into a voltage. This method gives an exact measure of the particle displacement in the trap site and is further discussed in Sec. 4.4. Electronic interference detection is now widely used as it gives an accurate description of the trapping potential and therefore a measure to compare trap stiffness for various particle sizes and materials. Additionally, Svoboda and Block employed direct imaging with video-enhanced differential-interference contrast (DIC) microscopy to observe the metal nanoparticles.

The ability to successfully control metal nanoparticles in three dimensions soon led to applications for touchless tweezing. Kawata et al. optically tweezed a single gold nanoparticle 

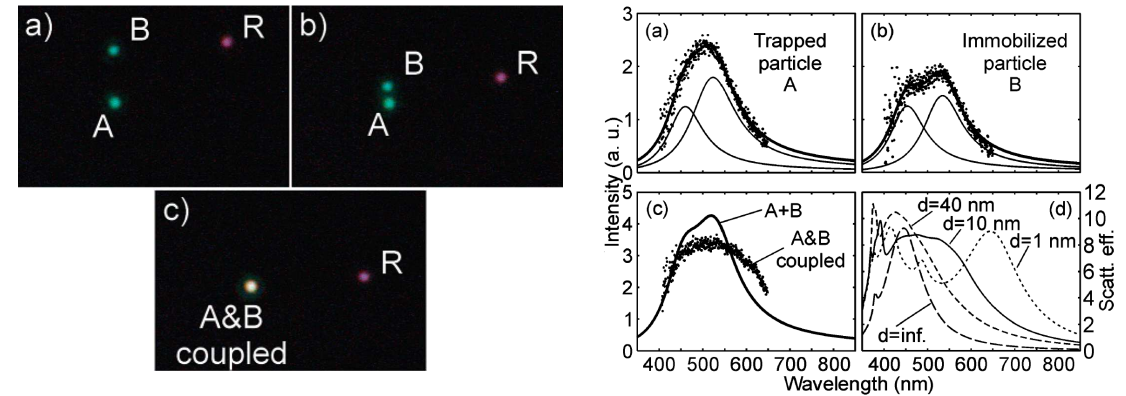

Fig. 4. Darkfield images of silver nanoparticles show the colourful plasmon excitation. The plasmon resonance shifts and broadens when two nanoparticles are combined to one larger particle by manipulating them in close proximity to one another. The change in resonance is visible by eye in the darkfield images and in the recorded scattering spectra. The solid lines are calculated fits to Lorentzian line shapes (thin lines). The spectrum of a coupled nanoparticle pair is very sensitive to the particle separation (d). Adapted from [58] and reprinted by permission from ACS Publications: Nano Letters, copyright (2004).

and used it as a near field scanning probe [59]. The advantage of this method is that the probe particle has no contact with any interface or tip. This contact-free SNOM probe may then be utilised for fluorescence or near field imaging.

Another possibility is to exploit optical tweezers as an experimental tool to investigate single particle properties. Prikulis et al. [58] tweezed a single silver nanoparticle, obtained its spectrum and demonstrated the resonance shift and broadening when two $90 \mathrm{~nm}$ silver particles interact in close proximity (Fig. 4). To monitor the trapped particle they used darkfield microscopy. In this configuration the numerical aperture (NA) of the trapping objective has to be smaller than the numerical aperture of the condenser objective in order to keep the darkfield microscopy working. This limited Prikulis et al. in their trapping abilities so that they were constrained to 2D trapping as the maximum used NA was 0.7. They also applied an indirect detection method, coupling the scattered light of the nanoparticle into a fibre spectrometer to analyse the particle's distinct spectrum. This gives a detailed insight into the particle's properties and allows determining the material and size of the metal nanoparticle. The position of the plasmon resonance of a Rayleigh particle of a certain material can easily be predicted. This resonance position shifts to the red for larger particles. Additionally Prikulis et al. demonstrated that two particles in close proximity show a very distinct scattering pattern as they may be combined of the scattering spectra of the individual particles. Soon after, pairs of $40 \mathrm{~nm}$ silver spheres were combined to form hot pairs for surface-enhanced Raman Spectroscopy in the focus of optical tweezers [60].

Recently Lene Oddershede's group started to conduct very detailed studies pushing the understanding of tweezing metal nanoparticles even further [61,62]. A wide size range of gold and silver nanoparticles has been investigated as well as a study to optimise optical tweezing of metal nanoparticles with respect to an aberration-compensated focus [63]. They examined the trapping potential for various sizes analysing the scattered light of a trapped particle with a quadrant photodiode. Here, the trapping laser is the probe laser at the same time. The interference pattern of the incoming trapping beam and the scattered light of the nanoparticle fall on the four quadrants of the diode and the displacement of the nanoparticle can be monitored with nanometre precision. Particles larger than $70 \mathrm{~nm}$ have been observed in brightfield microscopy. Below this diameter differential interference contrast microscopy (see Sec. 4.4) had to be applied to render the nanoparticles visible. They also mentioned that water immersion objectives are more effective than oil immersion for metal nanoparticle trapping. However, changing the refractive index of the immersion oil and position of the trap focus also proved beneficial for increasing the trap stiffness $\kappa$ as shown in Fig. 5. The group further demonstrated the im- 


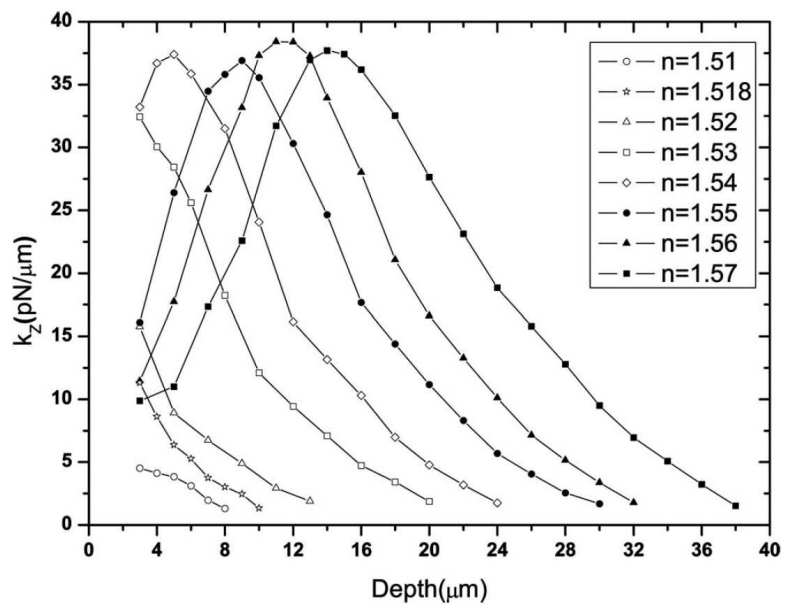

Fig. 5. Changing the refractive index $n$ of the immersion oil for the trapping objective increases the trap stiffness $\kappa$. The trapping potential in single-beam tweezers is harmonic and can be described as $F=-\kappa\left(x-x_{0}\right)$ with the trap stiffness $\kappa$, the equilibrium position of the particle $x_{0}$ and the position of the particle $x$. Increasing the trap stiffness $\kappa$ allows to use less laser power and still exert the same force on the particle. The correct choice of the position (depth) of the beam focus and thus the trapping position in the sample chamber is also important. Reprinted by permission from the Optical Society of America: Optics Letters [63], copyright (2007)

portance of aberration analysis in the optical trap site leading to tremendous improvements in trapping efficiency which is crucial for power reduction. This is essential to minimise heating and damaging effects for further applications.

The refractive index of metal nanoparticles contains a larger imaginary part compared to dielectric particles, representing the increased absorption in metal nanoparticles. This absorption potentially induces heating that may seriously affect the usefulness of tweezing metal nanoparticles for biological applications [64]. Seol et al. presented an experiment where they investigated the same optical trap with three different methods to determine the trap stiffness $\kappa$ based on the equipartition theorem, power spectrum and hydrodynamic drag. The discrepancies arising in these measurements were attributed to significant heating of the nanoparticle. When the nanoparticle heats up, the host medium within it is dispersed in changes its viscosity and therefore affects the power spectrum as well as the hydrodynamic drag. The viscosity of water decreases with increasing temperature and affects these two approaches that assume a constant viscosity. Seol et al. make a very valid point; however heating effects might not be as detrimental if an appropriate trapping geometry and wavelength is used for the specific application. Optimising the single-beam tweezers [63] such that minimal powers are required to trap nanoparticles is one approach. Alternative trapping geometries to trap metal nanoparticles out of high intensity regions provide an additional technique to avoid potential heating [65]. Further advances in plasmon based trapping may provide innovative means to decrease the amount of power necessary for optical trapping of metal nanoparticles.

Combining single-beam tweezers and evanescent wave trapping allowed Sasaki et al. to analyse the scattering force acting on $250 \mathrm{~nm}$ gold particles [66]. They created an evanescent field with a $1064 \mathrm{~nm}$ laser beam at one interface of the sample chamber by total internal reflection. The nanoparticle was manipulated close to the evanescent field with the help of a second beam shaped as standard single-beam tweezers (see Fig 6a). The gradient forces attracted the nanoparticle towards high intensity regions while the scattering force component of the evanescent field laterally displaced the nanoparticle. Although the particle was stably trapped in the 
a)

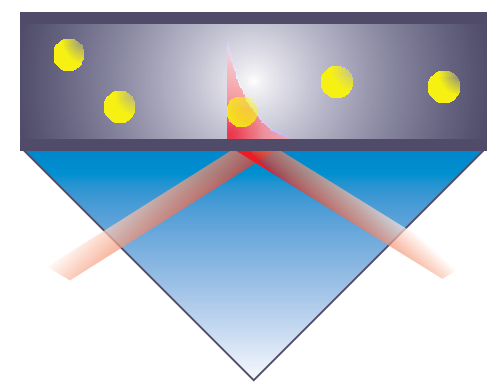

b)

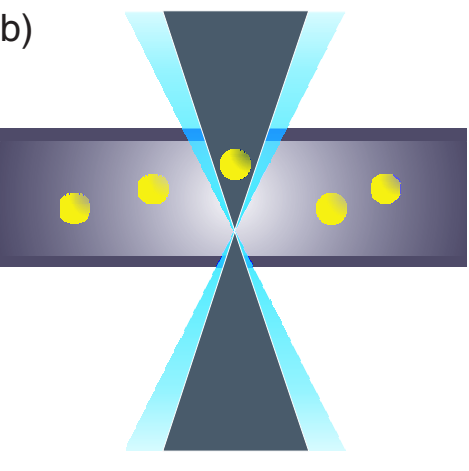

Fig. 6. a) Combining single-beam tweezers and evanescent fields lead to further insight in the interplay of scattering and gradient force components for metal nanoparticles [66]. b) A vortex trap is an alternative trapping geometry to single-beam tweezers [65]. The metal nanoparticle is confined in the dark vortex core of a Laguerre-Gaussian beam, avoiding the high intensity region of the laser.

single-beam tweezers, it could be further manipulated with the evanescent field. Sasaki $e t$ al. concluded that gradient force attraction is possible even for gold particles as large as $250 \mathrm{~nm}$ in a $1064 \mathrm{~nm}$ laser field. Further trapping geometries, for example the dual beam trap, have been suggested, but have not been realised for metal nanoparticles.

\subsubsection{Trapping near the plasmon resonance}

A particle plasmon is a resonant excitation of free electrons in the metal resulting in wavelength dependent scattering, absorption and polarisability of the nanoparticle. This resonant property influences the forces one may exert with an incident laser beam. The gradient force changes significantly with the wavelength dependent polarisability. The polarisability of a particle gives a measure how much charge separation can be acquired within the particle itself. For atoms or certain nanoparticles the polarisability may even become negative and thus lead to a repulsive gradient force. Close to the particle's resonance scattering and absorption also increase considerably. Consequently there has to be a well chosen balance of counteracting forces that compete in an optical trap to achieve the best possible trapping.

Theoretical investigations approaching the plasmon resonance for optical trapping suggested a wavelength dependent interaction process leading to a repulsive or attractive gradient force [33,67]. The sign of the gradient force is directly related to the sign of the particle's polarisability $\alpha$ which changes for certain metal nanoparticles close to resonance. This idea was theoretically studied further within the context of an evanescent wave trapping geometry [68]. Only within the last two years, experimental exploration of tweezing nanoparticles close to their resonance has gained momentum.

The first experiment investigated gold nanorods with the longitudinal resonance around 800 nm [69]. Pelton et al. showed repulsion of gold nanorods by a trapping laser tuned below the resonance wavelength and attraction into the trapping region for a trapping laser tuned above the resonance. Further trapping of bipyramids and Au-Ag core-shell nanorods strengthened the observation of an enhanced gradient force at the red-detuned side of the nanoparticle's resonance [70]. In both experiments a tuneable Ti:Sapphire laser served as trapping laser and a second modelocked laser at $800 \mathrm{~nm}$ with a tenth of the power of the trapping laser induced two photon fluorescence of the nanorods. This fluorescence was analysed with a photodiode recording residence times of the nanorods in the trap (see Fig. 12 and Sec. 4.4). Pelton et al. observed repulsion of the gold nanorods from the trap site when the laser was tuned below resonance resulting in a shorter residence time in the trap site. Using a laser wavelength above 
the resonance of the gold nanorods, so-called red-detuning of the trapping laser, resulted in an increase in trap residence time of the nanorods.

Non-spherical metal nanoparticles are complex structures sustaining more than one plasmonic resonance mode. Nanorods for example have two resonances corresponding to the long and short axis excitation. The long-axis resonance is red-shifted compared to the resonance of a spherical particle of equivalent dimensions. This red-shifting of the resonance is controlled with the aspect ratio (length over thickness) of the nanorod. Furthermore, the long axis plasmon excitation exhibits lower nonradiative damping at resonance. The interband damping mechanism is suppressed as the lower (red-shifted) resonance energy does not exceed the necessary threshold energy to excite interband transitions [71]. Interband damping is a nonradiative decay of the particle plasmon by creating excitons (electron-hole pairs). Contrary to plasmon resonances in nanospheres, the excitation of particle plasmons in non-spherical objects depends on the orientation of the nanoparticle with respect to the polarisation of the trapping laser.

Close to the plasmon resonance the absorption cross section of a nanoparticle increases and is likely to induce heating as discussed in Sec. 3.2.1. To keep the heating effect at a minimum one may optimise the power requirements for single-beam tweezers or consider different methods of optical trapping. An alternative trapping geometry, commonly used in atom trapping, relies on the repulsive nature of the trapping laser [65]. The metal nanoparticles are repelled out of the high intensity regions of the trapping laser rather than being attracted to the focus (see Fig. 6b). This repulsion may either originate from a negative gradient force or a strong scattering force component. Both processes occur at the blue-detuned side of the resonance of a nanoparticle. Depending upon the specific material properties either of these two processes may dominate. A single ringed (radial index $p=0$ ) Laguerre-Gaussian (LG) beam generates an annular trapping laser profile (zero intensity in the core). Once loaded into the trap site the particles are surrounded by the high intensity region of the beam. Choosing the appropriate laser wavelength with respect to the particle plasmon resonance is crucial for resonant optical trapping and we are going to discuss this further in Sec. 4.3.

\subsection{Trapping molecules}

Indirect trapping of single molecules, DNA in particular, has been extensively investigated [7274]. Here the molecule is attached with suitable chemistry to a micron-sized dielectric bead. Instead of exerting optical forces directly on the molecule, the dielectric bead is trapped and easily manipulated with single-beam tweezers. The gradient force holding an object in the trap is directly dependent on the polarisability $\alpha$ of the particle and therefore its volume; decreasing for smaller nanoparticles and increasing for larger micron-sized beads. The main difficulty is the submicron size of the molecules and thus the decreased forces a trapping laser can possibly exert on a single molecule. Applying higher trapping laser powers and experimenting with the resonance of fluorescent labels provided the means to achieve direct trapping of molecules during the past decade [75-78].

Compact DNA as in a globular or supercoiled state is denser and therefore easier to tweeze than elongated molecules. Stable trapping for single DNA molecules has been reported at laser powers around $200 \mathrm{~mW}$ using red or infrared trapping lasers [75, 76]. As there is less biological damage from a $1064 \mathrm{~nm}$ laser, increasing the beam power up to $500 \mathrm{~mW}$ enables trapping of simple coiled (elongated) DNA molecules as pictured in Fig. 7 [77]. These do shrink in the trap but not as much as in a supercoiled state. In all described experiments the trapping mechanism is attributed to larger gradient forces for the more compact molecular structure. In these experiments the DNA was labelled with a fluorescent dye to observe it with a SiliconIntensified Target (SIT) camera. Analysing the images of the fluorescent dyes provided the information needed for data acquisition.

Another trapping mechanism has been suggested, taking the role of the fluorescent labels into account. The attached fluorophores have their own distinct resonance, resulting in an 

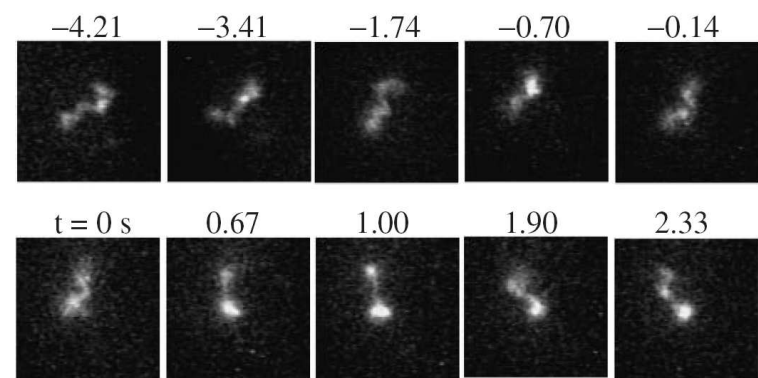

1.90


2.33

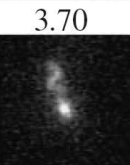

5.04

6.37

7.24
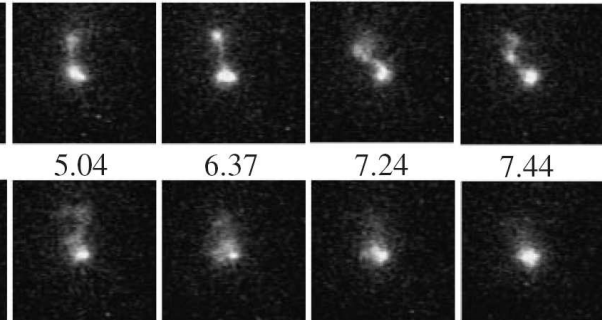

7.44

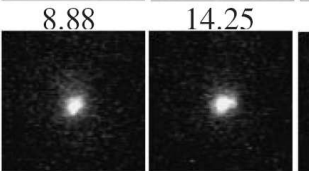

14.65
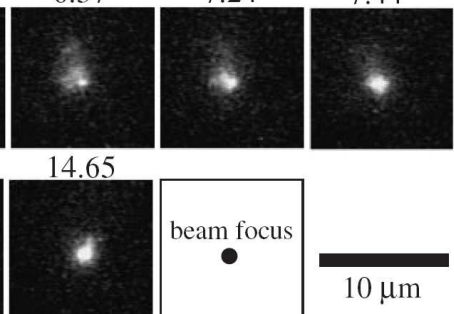

$10 \mu \mathrm{m}$

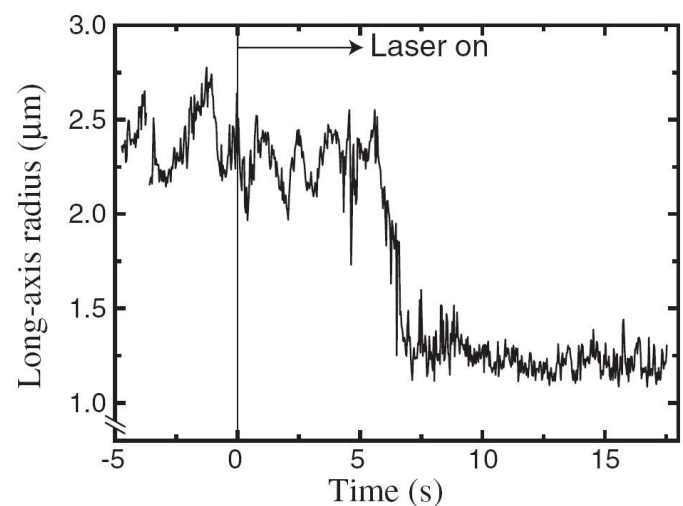

Fig. 7. a) Direct optical trapping of a coiled T4 DNA molecule imaged in fluorescent microscopy. The free molecule fluctuates due to Brownian motion and once the laser is switched on $(t=0)$ the coil is pulled into the trapping laser focus. b) The radius of the DNA fluorescent image decreases over time and thus represents the accumulation of the entire molecule in the laser focus. Reprinted by permission from the Physical Society of Japan: Journal of the Physical Society of Japan [77], copyright (2005).

increased polarisability and therefore enhanced gradient forces at the fluorophore's resonant wavelength. These labels are essential in visualising and detecting single molecules. The fluorescent labels are either excited directly by the trapping laser or with a second probe laser. Recent investigations showed that the trapping efficiency depends upon the number of fluorophores attached to a single antibody [78]. Li et al. found clear evidence that the resonant excitation of these labels enables trapping for single antibodies up to $1 \mathrm{~s}$ at powers as low as $0.75 \mathrm{~mW}$. The exact mechanism remains to be explored as the resonance effect shows molecule attraction into high intensity region of the trapping laser even for blue-detuned traps. Li et al. conclude that the dipolar model employed for the attached fluorophores might not be appropriate.

In addition to direct optical trapping of single molecules several groups observed biased diffusion of molecules into a high intensity region of a laser beam [78-81]. The diffusion 
statistics of the molecules do not follow the expected Poisson statistics at short time scales. This process suggests that the molecules are attracted towards regions of high laser intensity. The laser intensities applied in these diffusion experiments are with 10-31 $\mathrm{mW}$ generally lower than in the trapping experiments. Osborne et al. discuss resonant and non-resonant contributions to the biased diffusion of single molecules [79].

The most common data acquisition method when trapping molecules is to count the photon bursts of the fluorescence. The fluorescence signal from the trap site is focussed on a photomultiplier or avalanche photodiode that are coupled to photon counting units. This gives time dependent photon counts providing fluorescence spectra that are further analysed with Poisson or autocorrelation statistics. Here, the short time scale analysis is in particular important as the trapping expresses itself as an increase in photon burst during a very short timescale while the molecule is hold in the trap site. A different method of analysis was performed by Singer et al. who used a helium neon laser at $633 \mathrm{~nm}$ as probe beam [81]. Depending on the content of the trap, the beam was scattered in a distinct manner. By blocking the central part of the beam and only analysing the rays scattered at angles larger than the incoming beam, the sensitivity was increased even further. The scattering pattern was recorded with a CCD camera and processed with Matlab software analysing the intensity changes.

\section{OPTICAL TWEEZERS: OPERATION AND CONSTRUCTION}

Optical tweezers is an established method for manipulating single microscopic particles in three dimensions. The assembly of an optical tweezers system involves some important governing principles and due consideration of certain mechanical components. A more comprehensive technical discussion of this may be found elsewhere [82] but here we give the reader an overview of the issues involved. Many considerations when assembling any optical trapping system are based upon choice of numerical aperture of the objective, the types of lasers and the beam delivery which are pertinent to the design. In particular, with relevance to this paper, one major challenge when experimenting with nanoparticles is their visualisation and detection. There are various methods where nanoparticles can be observed directly. We focus initially upon a generic optical trap but will concentrate in following sections upon how we achieve successful trapping for nanoparticles with particular choices of objectives and lasers for that particular particle size. Subsequently, we address different methods of nanoparticle imaging and detection. We complete this experimental section with a discussion about measuring optical forces.

\subsection{Single-beam tweezers}

Full control of the position and form of a single laser beam is core to successful optical tweezers. Such a system may be readily implemented around a standard microscope which already comes with a high numerical aperture (NA) objective lens [82]. The standard microscope body adds robustness to the system and provides very high quality optics for a tweezers platform. Alternatively one may build the optical tweezers from standard cage plate or other opto-mechanical components. This offers an inherent flexibility and adaptability for optical trapping and may lead to more compact geometries. In relation to nanoparticle trapping, this may be achieved either on a microscope with suitable imaging or with a cage-plate system. Figure 8 illustrates a diagram of a standard tweezers setup.

In general, nanoparticles and molecules are dispersed in aqueous solutions as most of them are not stable when isolated. A practical reason is to damp the particle motion thus decreasing the Brownian motion of the nanoparticles. Dispersed particles at all size scales typically exhibit overdamped motion within the trap. Also, they are mostly small enough to float throughout the entire sample chamber instead of sinking to the bottom of the sample. This removes the requirement of additional beams for lifting the particles back up and manoeuvring them in the right trapping position as one can rely on diffusion of the nanoparticles into the trap site 


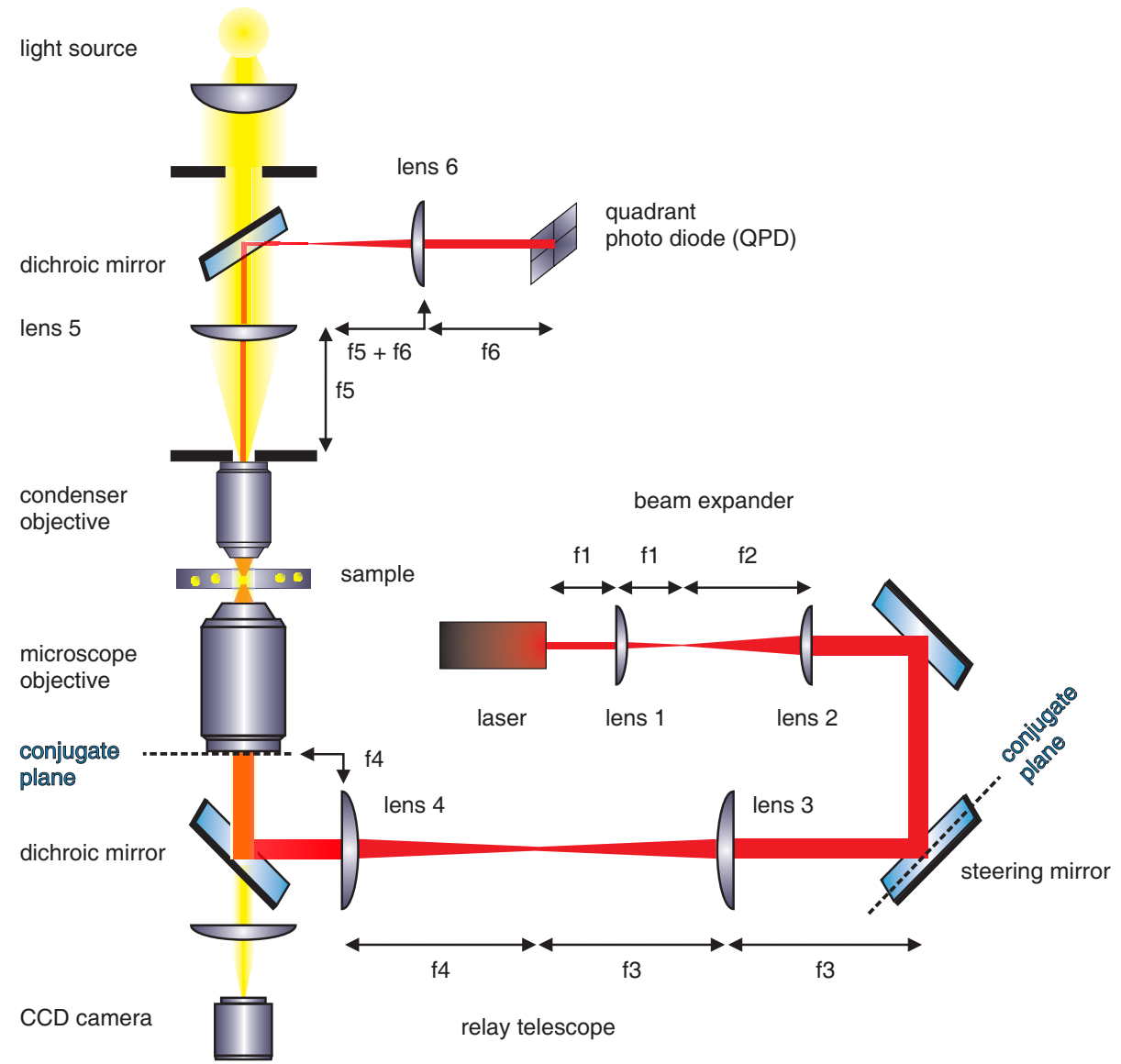

Fig. 8. To trap nanoparticles the standard optical tweezers have to be set up carefully to achieve the highest quality trap and imaging. The focal lengths of the lenses are labelled accordingly (e.g.lens 1 with a focal length $\mathrm{f} 1$ ). The beam expander, relay telescope and the steering mirror make sure that the back aperture of the high NA trapping objective is slightly overfilled with the laser beam. This is essential for good axial (z) trapping. Additional care has to be taken for setting up the illumination. Shown here is brightfield Koehler illumination. Direct imaging of the sample is possible with a CCD camera, whereas indirect observation and precise measurement requires a quadrant photodiode.

because of Brownian motion. In practice, approximately 10 microliters of solution is placed on a sample chamber which is typically around $1 \mathrm{~cm}$ in diameter and 100 microns deep. In the case of biological cells, these are often suspended in suitable buffer solution. The sample chamber consists of two glass coverslips comprising the sample and separated with a thin spacer, for instance adhesive stickers, vacuum grease or nail varnish, which determines the height of the sample chamber and seals it to prevent the sample from drying up.

The sample is inserted into the sample plane of the optical tweezers which is situated at the focus of the trapping microscope objective (see Fig. 8). It is either illuminated from the top (inverted microscope) or from the bottom (upright microscope) depending upon the type of microscope setup. The various methods to illuminate and image the sample are discussed in Sec. 4.4. For nanoparticle imaging, the condenser lens ideally consists of a microscope objective to have a larger NA than a lens increasing the total resolution of the system. In transmission microscopy the trapping laser is inserted in the sample opposing the illumination 


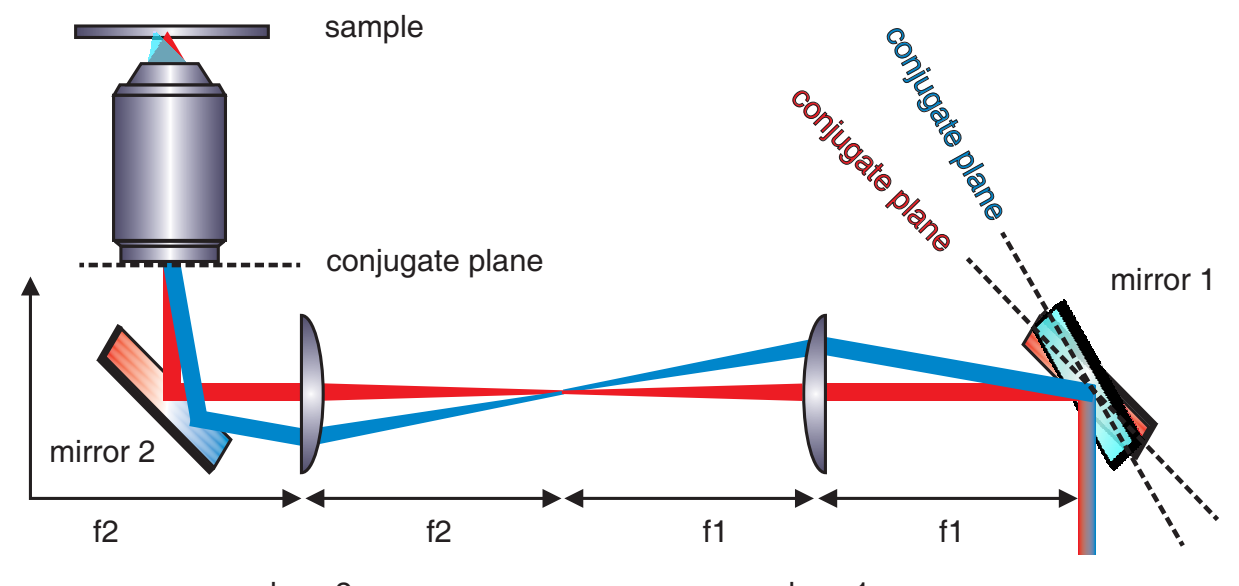

lens 2

lens 1

Fig. 9. Setting up the beam steering mirror mirror 1 at the conjugate plane of the back aperture of the trapping objective assures that the beam can be manipulated without walking of the back aperture of the objective. This allows moving the trapping position of the beam in the sample plane. At the same time the trapping laser always slightly overfills the back aperture of the microscope objective ensuring maximal axial as well as lateral trapping strength.

beam through a high NA microscope objective. The trapping and illumination objective are fixed, only the sample stage and the laser beam inside the objective can be moved. Optical conjugate planes and the appropriate telescope are used to steer the beam inside the objective in all three dimensions.

Optical conjugates enable the optimum steering of the trapping beam before entering the microscope objective. The input beam needs to be translated across the sample stage whilst ensuring the beam does not "walk off" or is deviated in a way that might cause it to miss or clip any apertures in the beam path, most notably the back aperture of the microscope objective. Ensuring that the input beam is centred upon the back aperture during steering ensures a good quality trapping beam at the focal plane at all times. It is also important to eliminate any offaxis aberrations that might be present, arising from the misalignment in the optical train, for example astigmatism and coma.

In Fig. 9 we show how to implement the idea of optical conjugates for the steering of the trapping beam. The back aperture of the microscope objective is imaged onto a mirror. Thus positioning the light onto this mirror is equivalent to positioning the beam through the objective: tilting the mirror in the lateral plane now moves the beam at the sample plane but does not move it across the back aperture of the microscope objective. The beam steering system is composed of two lenses, lens 1 (focal length $=f 1$ ) and lens 2 (focal length $=f 2$ ), and a steering mirror mirror 1. The lenses are spaced at the sum of their focal lengths and mirror 1 is positioned at one focal length $f 1$ away from lens 1 . By placing the back aperture of a microscope objective at one focal length $f 2$ away from lens 2, a beam steering lens system is formed. Here, the rotating mirror 1 and the back aperture of the microscope objective form optical conjugates. Both the blue line (deflected beam) and the red line illustrate the rays from rotating mirror 1 . The position of the central rays is maintained at the back aperture of the microscope objective.

\subsection{Choice of objective}

A key goal for a powerful optical tweezers system is to attain a strong trap stiffness which ultimately means a tightly focused beam spot. To achieve a diffraction-limited beam spot with the laser at the focal plane, a high numerical aperture (min NA $\approx 1$ ) microscope objective lens is a natural choice. Today, microscope objectives can produce an image at infinity. With so-termed 
"infinity corrected" objectives, researchers may use additional telescoping lenses and optical conjugates to relay the collimated laser source to the microscope objective (within the "infinity space") without affecting the bright-field or fluorescence imaging aspect of the system. The optical telescope is desirable for achieving a diffraction limited spot for true three dimensional tweezing as it assists in slightly overfilling the back aperture of the microscope objective [82].

When employing an oil-immersion microscope objective for tweezing, the beam propagates through a layer of index matching oil through a thin piece of glass (the coverslip) and then into the sample solution which is of a lower refractive index than the glass and oil. The beam will undergo a large change in refractive index that imposes spherical aberrations and results in broadening of the beam spot along the axial plane. Thus, a well aligned optical tweezers may exhibit inherent aberrations due to refractive index mismatches that can result in poor trapping particularly at depth. As the result, the trap performance may degrade when tweezing deeper into the sample [29]. Another method is to use water immersion objectives for optical trapping of nanoparticles. They are corrected for the refractive index of water and, by removing the oil and coverslip, are inserted directly into the sample which reduces the spherical aberration. As discussed in Sec. 3.2.1 Reihani et al. conducted a detailed study about aberrations and trap focus position [63]. They demonstrated that oil immersion objectives, when used in combination with the correct index matching liquid, are potentially more suitable to trap metal nanoparticles than water immersion objectives.

Other optical techniques to obviate these aberrations are available in the form of dynamic holographic [83] elements or a deformable mirror [84] technology by imposing correction terms of the appropriate Zernike polynomials upon the input wavefront of the tweezing beam.

\subsection{Laser choice}

The choice of laser has a major influence upon optical trapping. This is not only in terms of the wavelength deployed but also the mode of laser operation i.e. continuous wave or mode locked (short pulse). Most optical trapping experiments use single wavelength continuous wave (CW) sources. For large biological objects it is important to avoid high absorption of the light by the subject being trapped. The laser wavelength is chosen within the so-termed therapeutic window in the near-infra red region to avoid damage to biological specimens. In more detailed studies, wavelengths of $830 \mathrm{~nm}$ and $970 \mathrm{~nm}$ have been shown to be particularly favourable [85]. The authors explored the wavelength dependent nature of the photodamage in E. Coli compared to Chinese hamster ovary $(\mathrm{CHO})$ cells. The cloning efficiency of $\mathrm{CHO}$ cells after irradiating for 5 minutes was studied and the wavelengths $830 \mathrm{~nm}$ and $970 \mathrm{~nm}$ were observed to offer highest efficiency (and thus optimal cell viability), whilst the wavelength range from $870 \mathrm{~nm}-930$ $\mathrm{nm}$ was found to be the most damaging. More generic studies quite often use wavelengths at $\approx 1064 \mathrm{~nm}$ due to the ease of obtaining very high quality fibre laser sources. One also has to consider the impact upon the heating of the surrounding medium which can be detrimental in such experiments.

In the near infra red, there are ample laser sources with good beam shape that is typically characterized by the $\mathrm{M}^{2}$ parameter: this denotes how close an output beam is to a perfect Gaussian beam profile ( $\mathrm{M}^{2}=1$ for a single-mode $\mathrm{TEM}_{0}$ Gaussian beam) [86]. Additionally beam pointing stability is a major issue: motion of the beam here can radically alter any detailed force measurements. For studies focused upon colloidal science, high power lasers in the visible range of the spectrum (notably at $532 \mathrm{~nm}$ ) are favourable due to the low absorption of water that reduces heating effects. Ultra-short pulsed lasers have also been used when one wishes to combine optical trapping with nonlinear phenomena (two photon excitation). If the repetition rate of the laser is high enough, anomalies due to particle diffusion in between pulses is not significant and the forces exerted equal to their continuous wave counterparts [87]. Short pulsed lasers have also been used for visualisation of trapped nanoparticles [69]. 

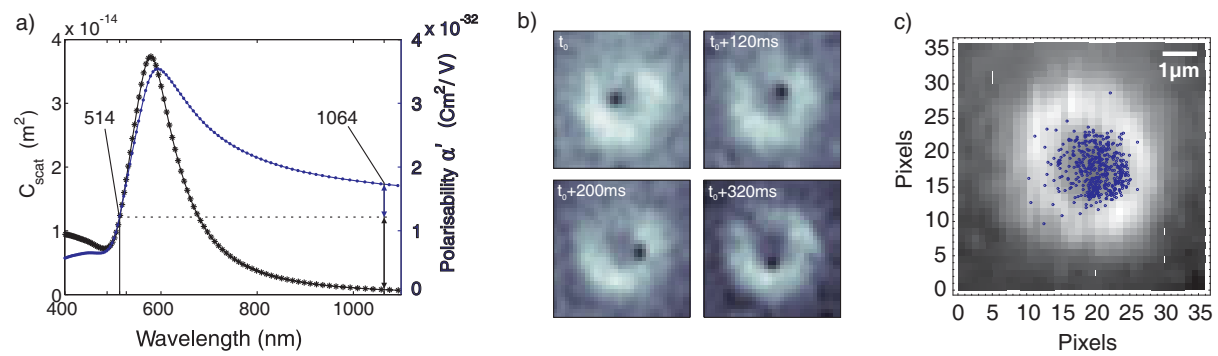

Fig. 10. The particle plasmon resonance results in a wavelength dependence of the optical forces exerted with a laser on metal nanoparticles. a) As an example we calculated the polarisability $\alpha$ and the scattering cross section of a $100 \mathrm{~nm}$ gold sphere. Compared to the typical trapping wavelength at $1064 \mathrm{~nm}, \alpha$ is decreased for $514 \mathrm{~nm}$. Contrary, the scattering cross section is increased at $514 \mathrm{~nm}$. A $514 \mathrm{~nm}$ laser thus exerts less attractive force than a $1064 \mathrm{~nm}$ laser and pushes the nanoparticles out of high intensity regions because of the increased scattering force components. b) Instead of manipulating $100 \mathrm{~nm}$ gold particles with single-beam tweezers we show how one may confine the nanoparticles to a dark vortex core of a $514 \mathrm{~nm}$ Laguerre-Gaussian beam. The particle remains in the dark core as shown in the movie stills of this process. The video data is further analysed with particle tracking and in c) we plot all positions one nanoparticle visited during confinement.

Whilst spatial coherence of the light source is crucial for a tightly focused beam, temporal coherence is not such an issue (except of course where the laser bandwidth encroaches upon an absorption band within the trapped sample). Some recent experiments have even used "white light" laser sources based on a supercontinuum generation [88]. Such a source offers the potential for spectroscopy simultaneously with the trapping and the prospect of tuning around the plasmon resonance.

Metal nanoparticles, as well as other particles exhibiting a resonance, require extra thought for choosing the trapping laser wavelength. A resonant effect can only be induced with a laser wavelength close the resonance wavelength of the particle. Here, attractive gradient forces may be reduced or even turned into repulsive gradient forces. Additionally, scattering and absorption are likely to increase. In order to exploit the particle plasmon resonance for optical trapping, the selected trapping geometry has to work in favour of the induced optical forces.

The resonantly induced change in optical forces may be explained in the quasi static limit where the total force acting on the particle consists of a dipolar gradient force as well as scattering and absorption forces as we discussed earlier in Sec. 2.3. The latter forces depend linearly upon the scattering and absorption cross section of the nanoparticle. The gradient force depends linearly upon the polarisability $\alpha$ of the particle. The polarisability gives a measure of the amount of charge separation the electric field induces in the metal nanoparticle. In Fig. 10a we plot the polarisability for a $100 \mathrm{~nm}$ gold sphere. The polarisability $\alpha$ and thus the gradient force is reduced below resonance when comparing $\alpha$ at $1064 \mathrm{~nm}$ (a wavelength far off resonance) and $514 \mathrm{~nm}$ (a wavelength on the blue-detuned side of the resonance). At the same time, the scattering cross section and therefore the scattering force increases for $514 \mathrm{~nm}$ compared to $1064 \mathrm{~nm}$ laser excitation. Consequently the balance between scattering and gradient forces that enable optical trapping at $1064 \mathrm{~nm}$ is destabilised close to resonance. It turns out to be impossible to tweeze $100 \mathrm{~nm}$ gold spheres in the high intensity region of a $514 \mathrm{~nm}$ laser focus and one has to use an alternative trapping geometry to trap the particle.

A dark-field vortex trap is one alternative to single-beam tweezers, confining the nanoparticle to the dark core of a Laguerre-Gaussian beam [65]. The geometry is explained in Sec. 3.2.2 and the movie stills of the experiment are shown in Fig. 10b. The nanoparticle is enclosed by a high intensity region of laser light. The positions the nanoparticle residences are plotted in Fig. 10c. In the case of gold nanoparticles the repulsion out of the high intensity regions relies 
a)



b)

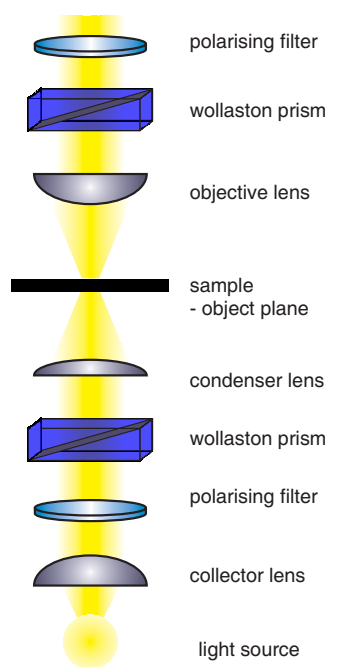

c)

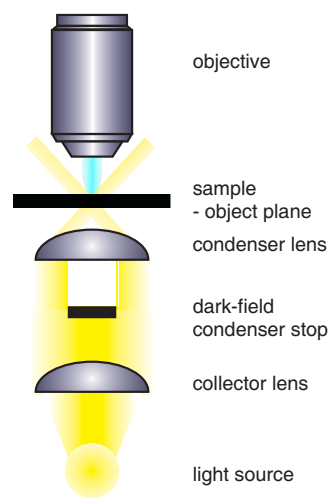

I

Fig. 11. A $100 \mathrm{~nm}$ gold sphere freely moving in solution imaged with Koehler illumination, DIC microscopy and transmission darkfield microscopy. a) In Koehler illumination the sample is uniformly illuminated with a bundle of parallel light rays at different angles. It is possible to image metal nanoparticles down to approximately $80 \mathrm{~nm}$ with this method. b) DIC is ideally applied to transparent samples but sometimes used for metal nanoparticles. c) The best solution appears to be darkfield imaging as it is possible to image particles much smaller than $80 \mathrm{~nm}$ without any additional labelling.

on resonantly enhanced scattering. However for other materials such as silver the repulsion is potentially the result of a negative gradient force when the polarisability becomes negative. This effect remains to be experimentally explored.

The described resonance detuning for trapping in a dark vortex core is widely used in atom trapping. The volume of atoms is extremely small and hence a standard gradient force trapping can not exert enough forces on atoms as the gradient force decreases with volume. The trapping laser is slightly detuned with respect to the atomic resonance. The resulting attractive or repulsive forces - red and blue detuned trapping respectively - are strong enough to manipulate atoms in three dimensions. The atomic resonance is much sharper as compared to the plasmonic resonance and thus requires only few nanometres of wavelength detuning of the trapping laser to change the forces exerted on atoms significantly.

\subsection{Imaging and detecting nanoparticles}

Direct imaging of nanoparticles faces two challenges: they are smaller than the diffraction limit and in some cases (dielectric nanoparticles) there is only little refractive index difference between the nanoparticles and the host medium. However, there are approaches to render nanoparticles visible that are frequently complemented with precise indirect detection techniques. We are going to illustrate selected methods that have proved useful and subsequently describe indirect measurement techniques.

Metal nanoparticles have a large refractive index and hence are easier to image in brightfield illumination than their dielectric counterparts of the same size. Standard brightfield microscopy requires Koehler illumination [89] to achieve the best possible resolution and contrast by evenly 



Fig. 12. a) The induced two-photon fluorescence has not been recorded with a CCD but with a spectrometer and compared to the extinction spectrum to really show that two-photon fluorescence was observed. b) Transmission-electron-microscopy or scanning electron microscopy is a very nice tool to analyse fine features of nanoparticles. This is only possible once they are immobilised on a conducting substrate. The time trace of the two-photon fluorescence in c) confirms when particles are in the trap. Reprinted by permission from the Optical Society of America: Optics Letters [69], copyright (2006).

illuminating the field of view. With this method it is possible to image metal nanoparticles down to a size of approximately $80 \mathrm{~nm}$ (Fig. 11a). Differential interference contrast microscopy (DIC) is a technique for even smaller or transparent dielectric nanoparticles. DIC requires polarised light and two Wollaston prisms inserted in the illumination path [89]. These separate and rejoin the two differently polarised illuminating rays before and after the object. The polarised rays do not pass the sample in the exact same position and thus acquire a phase difference relative to one another as they travel through differently optically dense media. Bringing the rays into interference after passing through the sample shows the phase difference by creating a highcontrast image (Fig. 11b).

Darkfield microscopy is complementary to the previously discussed methods. The incident illumination merely passes the sample at a glancing angle and misses the observation objective. This objective only collects the scattered light of the nanoparticles that appear at high contrast in front of a dark background [89]. Darkfield microscopy is particularly useful when the nanoparticles show certain resonance effects such as particle plasmons or exciton resonances in semiconductors (Fig. 11c). However, combining darkfield microscopy with optical trapping remains challenging. The sample is imaged through the same objective which is used for optical trapping. The numerical aperture of the imaging/trapping objective has to remain smaller than the numerical aperture of the darkfield condenser. This requirement limits the combination of possible objectives. Optical trapping requires high numerical aperture oil or water immersion objectives to achieve stable 3D tweezing of nanoparticles. Consequently the condenser objective has to be an oil immersion objective in order to attain a higher numerical aperture. A different approach is to use reflected light darkfield microscopy. This requires a special objective (labelled as Neo, BF/DF, or BD depending upon the manufacturer) in combination with a block in the illumination path and a ring-shaped mirror. This mirror reflects a cylinder of light into the 
objective where the illuminating light is guided in an outer hollow collar whereas the trapping laser and the collected reflected light pass through the inner core of the objective [90,91].

An alternative imaging technique uses a secondary beam that excites two-photon fluorescence in the nanoparticles. These signals are shifted in frequency compared to the scattered light of the nanoparticles and thus are easy to separate from any background radiation (Fig. 12a). The two-photon fluorescence is collected with a CCD camera and the recorded images or videos can then be further processed to analyse the particle's position and movement [69]. Another widely used method is fluorescent labelling of DNA or dielectric nanoparticles with fluorescent stains. There is a variety of very well developed microscopic techniques such as confocal fluorescence microscopy as well as far - and near field microscopy. Far-field imaging employs higher laser powers for sub-millisecond time resolution whereas near-field microscopy yields higher spatial resolution [79]. Fluorescent imaging is applied in a variety of conditions; however its applicability is sometimes constrained because of the limiting effects of photobleaching.

Instead of directly imaging the nanoparticles there are indirect techniques to determine their position and movement as discussed in Sec. 4.5. Nowadays the most common method is the interferometric position detection with a quadrant photodiode. Here an interference pattern of the trapping beam and the scattered light of the particle impinge onto the four quadrants of the photodiode where it is converted into an electric signal, mostly a voltage. Subtracting and adding the electronic signals of the four quadrants yields nanometre precise position data for the nanoparticle. It is possible to retrieve a power spectrum that gives valuable information about trap stiffness and trapping potential. A common method, mostly applied in molecular studies, is the use of an avalanche photodiode or photon counter (Fig. 12c). Here the residence times of particles in a trap are recorded and further analysed [80].

\subsection{Measuring optical forces}

In Section 3.2.1 we review experiments from Oddershede's group and others about optical trapping of metal nanoparticles that focus in particular on measuring trap stiffness and characterising the trap $[27,61,63]$. In this section, we discuss how the optical forces are derived from these experiments. Optical tweezers offer a very accurate force measurement system. This may be understood by considering the underlying physical principles of its operation. This knowledge is then combined with an understanding of the particle dynamics and Brownian motion. The fundamental tenet is that an optical tweezers may be considered as a highly overdamped simple harmonic oscillator. A parabolic potential well is created by the optical trapping beam within which the particle resides. When the particle is at equilibrium, it tends to rest at centre of the potential well (trap) where it has the minimum energy. Though it may be considered as in a metastable state, it can escape the potential well if the well depth is not very high. For any motion away from the equilibrium position, over a small distance from that equilibrium, the particle will experience a restoring force that is proportional to the distance away from centre of the well. Thus the trapped object obeys Hooke's law and is rather like a microscopic spring. Most importantly the particle position may be determined to dimensions one order of magnitude smaller than that of the trapping wavelength which is the crux of the reason why such small displacements (and thus forces) may be discerned with this method. Mathematically we represent this situation by a one dimensional equation of motion in direction $\mathrm{x}$ for a Brownian particle in a harmonic potential:

$$
m \frac{\partial^{2} x}{\partial t^{2}}+\gamma \frac{\partial x}{\partial t}+\kappa x=F_{f}(t)
$$

In this equation, the first term represents the inertial force component for a particle with mass $m$, the second term is the velocity dependent viscous damping force ( $\gamma$ is the drag coefficient) and the final term is the optical restoring force for the trap stiffness $\kappa$. The right hand side of the equation represents the fluctuating force $F_{f}(t)$ initiated by Brownian motion. Normal trapping 


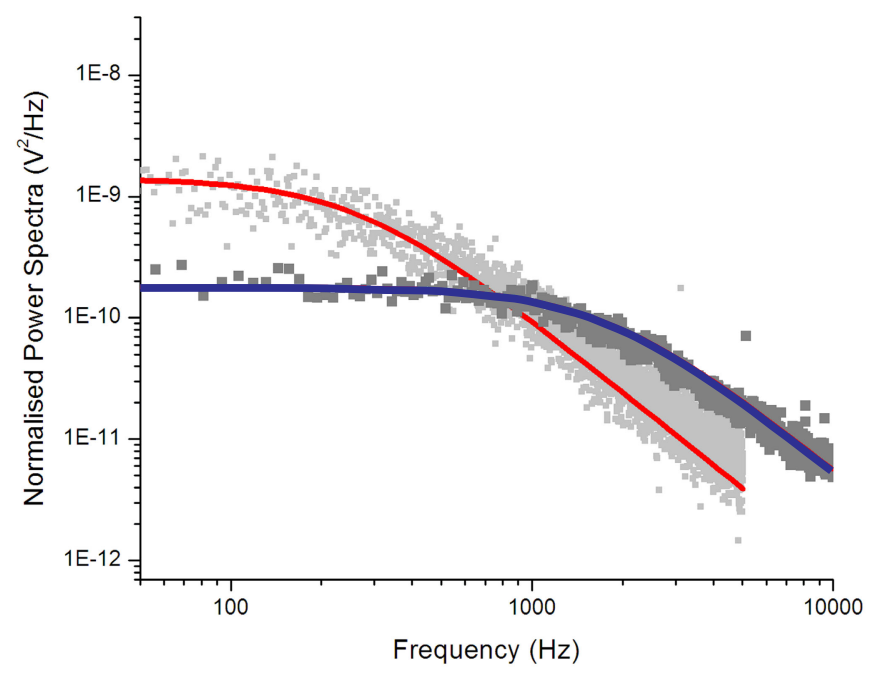

Fig. 13. The power spectrum of a trapped $100 \mathrm{~nm}$ gold (blue) and $100 \mathrm{~nm}$ polysterene (red) sphere can be fitted wit a Lorentzian curve. The curve fit provides the roll off frequency $f_{0}$ for each particle in at a given power in a specific optical trap. The roll off frequency determines the trap stiffness $\kappa$.

conditions in a sample medium with buffer means viscous forces dominate and we are in the low Reynolds number regime [92]. We may thus ignore inertial effects as $m \frac{\partial^{2} x}{\partial t^{2}} \ll\left(\gamma \frac{\partial x}{\partial t}+\kappa x\right)$ and the objects in our sample solution adhere to overdamped particle dynamics.

To use optical tweezers for measuring small forces, we require the addition of an optoelectronic sensor capable of measuring the mean position of the trapped object in all three dimensions and the corresponding trap stiffness $\kappa$. The sensor and detection circuitry is calibrated by moving the trapped objects over a known distance. With the relationship of signal (voltage) and the distance (nanometre), the sensor can be calibrated for specific distances. Once this position calibration factor and the stiffness $\kappa$ of the optical tweezers are quantitivity collected, the optical tweezers can then be implemented as a force transducer. The trap stiffness $\kappa$ is calculated from analysis of the thermal motion of the trapped object or by application of known viscous drag forces.

A popular method of detection is the imaging of the back focal plane interference pattern from the trapped object [93] onto an optoelectronic detector (quadrant photodiode or position sensitive detector) [94]. The signal can be collected from either back scattered light or forward scattered light with the aid of probe laser beam, in general the trapping beam itself $[95,96]$. The detector measures the geometrical centre of the trapped particle, in three dimensions, over a given time scale. The motion of a trapped nanoparticle is determined by the balance between the thermal Brownian motion and the tweezers trapping force. In addition to the position detection, the quadrant photodiode acquires the power spectral density for all three dimensions $\mathrm{x}, \mathrm{y}$ and $\mathrm{z}$. An example is plotted in Fig. 13. The power spectrum provides the corner or roll off frequency $f_{0}$ that is used to determine the trap stiffness $\kappa=f_{0} 2 \pi \gamma$. We refer the reader to the paper by Lee et al. [82] that explains in detail the assembly and calibration of an optical tweezers and the choices regarding the detection system.

Neuman and Block also make pertinent comments regarding the quadrant photodiode and position sensitive detector detection system [2]. A direct comparison of video and quadrant photodiode detector techniques has also been investigated by Keen et al. [97]. To measure the 
movement produced by single-protein conformational change we expect displacements in the nanometre range. In the domain of nanoparticles, the use of a quadrant photodiode for particle position monitoring and data accquisition to characterise trap properties is now an established method [61].

\subsection{Summary}

Trapping nanoparticles has advanced significantly over the past decade. It has evolved from some initial scepticism into a well established field. Optimisation of trapping geometries and parameters as well as better understanding of trapping processes between the Rayleigh and Mie regimes have helped to improve our understanding of optical forces at this size scale. There are multiple methods to model optical forces acting on nanoparticles. Within the Rayleigh approximation, the optical forces are decomposable into a gradient and a scattering force. As the size of the nanoparticle increases, this approach fails and one has to take higher order corrections into account. The Lorenz-Mie scattering theory and the T-matrix method deliver these necessary corrections. A direct numerical calculation of optical forces is also feasible by using, for example, finite elements methods and integrating either Maxwell's stress tensor or its derivatives over the nanoparticle volume and surface. This large choice of methods presents the perfect ground to study the transition between atoms and much larger optical objects where geometrical optics is applicable.

In many respects the trapping of nanometric objects is in its infancy: substantially more experiments remain to confirm theoretical predictions and forces on these objects at the nanoscale. Intrinsic resonance may assist greatly in imaging, obviating the need for tagging. Metal nanoparticles due to their strong response and tremendous field enhancements still need further investigation close to their resonance to make full use of controllability and employ them as nanometric sensors and antennas. Direct manipulation of macromolecules such as DNA has started to be successful. This opens up the opportunity for parallel studies of molecules with beam multiplexing, exploring their motion on optical landscapes, would greatly benefit this area. The future for nanoscale manipulation looks very bright.

\section{Acknowledgments}

The authors thank Woei Ming Lee for the power spectrum in Fig. 13. KD is a Royal Society Wolfson Research Merit Award Holder. MD acknowledges the financial support of the Stiftung der Deutschen Wirtschaft, sdw (Foundation of German Business) and SPIE. The authors thank the UK Engineering and Physical Sciences Research Council for funding.

\section{References}

[1] D. Grier, “A revolution in optical manipulation," Nature 424, 810-816 (2003) [doi:10.1038/nature01935].

[2] K. Neuman and S. Block, "Optical trapping," Rev. Sci. Instr. 75, 2787-2809 (2004) [doi:10.1063/1.1785844].

[3] K. Dholakia, P. Reece, and M. Gu, "Optical micromanipulation," Chem. Soc. Rev. 37, 42-55 (2008) [doi:10.1039/b512471a].

[4] G. Roosen and C. Imbert, "Optical levitation by means of 2 horizontal laser-beams theoretical and experimental-study," Phys. Lett. A 59, 6-8 (1976) [doi:10.1016/03759601(76)90333-9].

[5] A. Ashkin, "Forces of a single-beam gradient laser trap on a dielectric sphere in the ray optics regime," Biophys. J. 61, 569-582 (1992).

[6] R. Gussgard, T. Lindmo, and I. Brevik, "Calculation of the trapping force in a strongly focused laser beam,” J. Opt. Soc. Am. B 9, 1922-1930 (1992). 
[7] C. Granqvist and O. Hunderi, "Optical properties of ultrafine gold particles," Phys. Rev. B 16, 3513-3534 (1977) [doi:10.1103/PhysRevB.16.3513].

[8] S. Norrman, T. Andersson, C. Granqvist, and O. Hunderi, "Optical properties of discontinuous gold films," Phys. Rev. B 18, 674-695 (1978) [doi:10.1103/PhysRevB.18.674].

[9] P. B. Johnson and R. W. Christy, "Optical constants of the noble metals," Phys. Rev. B 6(12), 4370-4379 (1972) [doi:10.1103/PhysRevB.6.4370].

[10] B. Cooper, H. Ehrenreich, and H. Philipp, "Optical properties of noble metals. II," Phys. Rev. 138, A494-A507 (1965) [doi:10.1103/PhysRev.138.A494].

[11] A. Kawabata and R. Kubo, "Electronic properties of fine metallic particles. II. Plasma resonance absorption,” J. Phys. Soc. Jpn. 21, 1765-1772 (1966) [doi:10.1143/JPSJ.21.1765].

[12] L. Scaffardi and J. Tocho, "Size dependence of refractive index of gold nanoparticles," Nanotechnology 17, 1309-1315 (2006) [doi:10.1088/0957-4484/17/5/024].

[13] M. Ordal, L. L. Long, R. J. Bell, S. E. Bell, R. R. Bell, R. W. Alexander, and C. A. Ward, "Optical properties of the metals $\mathrm{Al}, \mathrm{Co}, \mathrm{Cu}, \mathrm{Au}, \mathrm{Fe}, \mathrm{Pb}, \mathrm{Ni}, \mathrm{Pd}, \mathrm{Pt}, \mathrm{Ag}$, Ti, and $\mathrm{W}$ in the infrared and far infrared," Appl. Opt. 22, 1099-1119 (1983).

[14] M. Ordal, R. J. Bell, R. W. Alexander, L. L. Long, and M. R. Querry, "Optical properties of fourteen metals in the infrared and far infrared: $\mathrm{Al}, \mathrm{Co}, \mathrm{Cu}, \mathrm{Au}, \mathrm{Fe}, \mathrm{Pb}, \mathrm{Mo}, \mathrm{Ni}, \mathrm{Pd}, \mathrm{Pt}$, Ag, Ti, V, and W," Appl. Opt. 24, 4493-4499 (1985).

[15] R. Cohen, G. Cody, M. Coutts, and B. Abeles, "Optical properties of granular silver and gold films," Phys. Rev. B 8, 3689-3701 (1973) [doi:10.1103/PhysRevB.8.3689].

[16] R. Jones, "Radiation pressure of light in a dispersive medium," Proc. R. Soc. London, Ser. A 360, 365-371 (1978).

[17] R. N. C. Pfeifer, T. A. Nieminen, N. R. Heckenberg, and H. Rubinsztein-Dunlop, “Colloquium: Momentum of an electromagnetic wave in dielectric media," Rev. Mod. Phys. 79, 1197-1216 (2007) [doi:10.1103/RevModPhys.79.1197].

[18] L. Novotny, R. X. Bian, and X. S. Xie, "Theory of nanometric optical tweezers," Phys. Rev. Lett. 79(4), 645-648 (1997) [doi:10.1103/PhysRevLett.79.645].

[19] P. C. Chaumet and M. Nieto-Vesperinas, "Electromagnetic force on a metallic particle in the presence of a dielectric surface," Phys. Rev. B 62(16), 11185-11191 (2000) [doi:10.1103/PhysRevB.62.11185].

[20] V. Yannopapas, “Optical forces near a plasmonic nanostructure," Phys. Rev. B 78(4), 45412 (2008) [doi:10.1103/PhysRevB.78.045412].

[21] Z. Li, M. Käll, and H. Xu, "Optical forces on interacting plasmonic nanoparticles in a focused gaussian beam,” Phys. Rev. B 77(8), 085412 (2008) [doi:10.1103/PhysRevB.77.085412].

[22] R. Loudon and S. Barnett, "Theory of the radiation pressure on dielectric slabs, prisms and single surfaces," Opt. Express 14, 11855 (2006) [doi:10.1364/OE.14.011855].

[23] M. Mansuripur, "Radiation pressure and the linear momentum of the electromagnetic field," Opt. Express 12, 5375-5401 (2004) [doi:10.1364/OPEX.12.005375].

[24] B. Kemp, T. Grzegorczyk, and J. Kong, "Lorentz force on dielectric and magnetic particles," J. Electromagn. Waves Appl. 20, 827-839 (2006) [doi:10.1163/156939306776143433].

[25] I. Brevik, "Experiments in phenomenological electrodynamics and the electromagnetic energy-momentum tensor," Phys. Rep. 52, 133-201 (1979) [doi:10.1016/03701573(79)90074-7].

[26] I. Brevik and R. Kluge, "Oscillations of a water droplet illuminated by a linearly polarized laser pulse,” J. Opt. Soc. Am. B 16, 976-985 (1999) [doi:10.1364/JOSAB.16.000976].

[27] K. Svoboda and S. Block, "Optical trapping of metallic Rayleigh particles," Opt. Lett. 19(13), 930-932 (1994). 
[28] R. Averitt, S. Westcott, and N. Halas, "Linear optical properties of gold nanoshells," J. Opt. Soc. Am. B 16, 1824-1832 (1999) [doi:10.1364/JOSAB.16.001824].

[29] A. Rohrbach and E. Stelzer, "Trapping forces, force constants, and potential depths for dielectric spheres in the presence of spherical aberrations," Appl. Opt. 41, 2494-2507 (2002) [doi:10.1364/AO.41.002494].

[30] J. Gordon, "Radiation forces and momenta in dielectric media," Phys. Rev. A 8, 14-21 (1973) [doi:10.1103/PhysRevA.8.14].

[31] B. Draine, "The discrete-dipole approximation and its application to interstellar graphite grains," Astrophys. J. 333, 848-872 (1988) [doi:10.1086/166795].

[32] P. Chaumet and M. Nieto-Vesperinas, "Time-averaged total force on a dipolar sphere in an electromagnetic field,” Opt. Lett. 25, 1065-1067 (2000) [doi:10.1364/OL.25.001065].

[33] R. Agayan, F. Gittes, R. Kopelman, and C. Schmidt, "Optical trapping near resonance absorption," Appl. Opt. 41(12), 2318-2327 (2002) [doi:10.1364/AO.41.002318].

[34] A. J. Devaney and E. Wolf, "Multipole expansions and plane-wave representations of electromagnetic-field," J. Math. Phys. 15, 234-244 (1974) [doi:10.1063/1.1666629].

[35] J. Barton, D. Alexander, and S. Schaub, "Theoretical determination of net radiation force and torque for a spherical particle illuminated by a focused laser beam," J. Appl. Phys. 66, 4594-4602 (1989) [doi:10.1063/1.343813].

[36] O. Farsund and B. Felderhof, "Force, torque, and absorbed energy for a body of arbitrary shape and constitution in an electromagnetic radiation field," Physica A 227, 108-130 (1996) [doi:10.1016/0378-4371(96)00009-X].

[37] J. Barton and D. Alexander, "Fifth-order corrected electromagnetic field components for a fundamental gaussian beam,” J. Appl. Phys. 66, 2800-2802 (1989) [doi:10.1063/1.344207].

[38] J. Lock, "Calculation of the radiation trapping force for laser tweezers by use of generalized Lorenz-Mie theory. II. On-axis trapping force," Appl. Opt. 43, 2545-2554 (2004) [doi:10.1364/AO.43.002545].

[39] J. Lock, "Calculation of the radiation trapping force for laser tweezers by use of generalized Lorenz-Mie theory. I. Localized model description of an on-axis tightly focused laser beam with spherical aberration,” Appl. Opt. 43, 2532-2544 (2004) [doi:10.1364/AO.43.002532].

[40] A. Mazolli, P. Neto, and H. Nussenzveig, "Theory of trapping forces in optical tweezers," Proc. R. Soc. London, Ser. A 459, 3021-3041 (2003).

[41] K. Ren, G. Grehan, and G. Gouesbet, "Prediction of reverse radiation pressure by generalized lorenz-mie theory," Appl. Opt. 35, 2702-2710 (1996).

[42] P. Waterman, "Symmetry, unity, and geometry in electromagnetic scattering," Phys. Rev. D 3, 825-839 (1971) [doi:10.1103/PhysRevD.3.825].

[43] M. Mishchenko, L. Travis, and A. Lacis, Scattering, Absorption, and Emission of Light by Small Particles, Cambridge University Publishing, Cambridge, United Kingdom (2002).

[44] M. Mishchenko, "Light scattering by randomly oriented axially symmetric particles," $J$. Opt. Soc. Am. A 8, 871-882 (1991).

[45] A. Ashkin, J. Dziedzic, J. Bjorkholm, and S. Chu, "Observation of a single-beam gradient force optical trap for dielectric particles," Opt. Lett. 11, 288-90 (1986).

[46] P. Zemánek, A. Jonáš, L. Šrámek, and M. Liška, "Optical trapping of nanoparticles and microparticles by a Gaussian standing wave," Opt. Lett. 24(21), 1448-1450 (1999) [doi:10.1364/OL.24.001448].

[47] S. Tan, H. Lopez, C. Cai, and Y. Zhang, "Optical trapping of single-walled carbon nanotubes," Nano Lett. 4, 1415-1419 (2004) [doi:10.1021/nl049347g]. 
[48] P. Pauzauskie, A. Radenovic, E. Trepagnier, H. Shroff, P. Yang, and J. Liphardt, "Optical trapping and integration of semiconductor nanowire assemblies in water," Nat. Mater. 5, 97-101 (2006) [doi:10.1038/nmat1563].

[49] K. Ajito and K. Torimitsu, "Single nanoparticle trapping using a Raman tweezers microscope," Appl. Spectrosc. 56(4), 541-544 (2002) [doi:10.1366/0003702021955015].

[50] J. Plewa, E. Tanner, D. Mueth, and D. Grier, "Processing carbon nanotubes with holographic optical tweezers," Opt. Express 12(9), 1978-1981 (2004) [doi:10.1364/OPEX.12.001978].

[51] J. Zhang, H. Kim, C. Oh, X. Sun, and H. Lee, "Multidimensional manipulation of carbon nanotube bundles with optical tweezers," Appl. Phys. Lett. 88, 053123 (2006) [doi:10.1063/1.2172020].

[52] A. van der Horst, A. Campbell, L. van Vugt, D. Vanmaekelbergh, M. Dogterom, and A. van Blaaderen, "Manipulating metal-oxide nanowires using counterpropagating optical line tweezers," Opt. Express 15(18), 11629-11639 (2007) [doi:10.1364/OE.15.011629].

[53] T. Yu, F. Cheong, and C. Sow, "The manipulation and assembly of CuO nanorods with line optical tweezers," Nanotechnology 15(12), 1732-1736 (2004) [doi:10.1088/09574484/15/12/005].

[54] R. Agarwal, K. Ladavac, Y. Roichman, G. Yu, C. Lieber, and D. Grier, "Manipulation and assembly of nanowires with holographic optical traps," Opt. Express 13(22), 8906-8912 (2005) [doi:10.1364/OPEX.13.008906].

[55] T. Iida and H. Ishihara, "Theoretical study of the optical manipulation of semiconductor nanoparticles under an excitonic resonance condition," Phys. Rev. Lett. 90(5), 57403 (2003) [doi:10.1103/PhysRevLett.90.057403].

[56] M. Pelton, "Comment on Theoretical study of the optical manipulation of semiconductor nanoparticles under an excitonic resonance condition," Phys. Rev. Lett. 92(8) (2004) [doi:10.1103/PhysRevLett.92.089701].

[57] A. Ashkin, "Applications of laser radiation pressure," Science 210(4474), 1081-1088 (1980) [doi:10.1126/science.210.4474.1081].

[58] J. Prikulis, F. Svedberg, M. Käll, J. Enger, K. Ramser, M. Goksor, and D. Hanstorp, “Optical spectroscopy of single trapped metal nanoparticles in solution," Nano Lett. 4(1), 115118 (2004) [doi:10.1021/n10349606].

[59] S. Kawata, Y. Inouye, and T. Sugiura, "Near-field scanning optical microscope with a laser trapped probe," Jpn. J. Appl. Phys 33, 1725-1727 (1994) [doi:10.1143/JJAP.33.L1725].

[60] F. Svedberg, Z. Li, H. Xu, and M. Käll, "Creating hot nanoparticle pairs for surfaceenhanced Raman spectroscopy through optical manipulation," Nano Lett. 6, 2639-2641 (2006) [doi:10.1021/nl062101m].

[61] P. Hansen, V. Bhatia, N. Harrit, and L. Oddershede, "Expanding the optical trapping range of gold nanoparticles," Nano Lett. 5(10), 1937-1942 (2005) [doi:10.1021/nl051289r].

[62] L. Bosanac, T. Aabo, P. Bendix, and L. Oddershede, "Efficient optical trapping and visualization of silver nanoparticles," Nano Lett. 8(5), 1486-1491 (2008) [doi:101021/nl080490+].

[63] S. Reihani and L. Oddershede, "Optimizing immersion media refractive index improves optical trapping by compensating spherical aberrations," Opt. Lett. 32(14), 1998-2000 (2007) [doi:10.1364/OL.32.001998].

[64] Y. Seol, A. Carpenter, and T. Perkins, "Gold nanoparticles: enhanced optical trapping and sensitivity coupled with significant heating," Opt. Lett. 31(16), 2429-2431 (2006) [doi:10.1364/OL.31.002429]. 
[65] M. Dienerowitz, M. Mazilu, P. Reece, T. Krauss, and K. Dholakia, "Optical vortex trap for resonant confinement of metal nanoparticles," Opt. Express 16(7), 4991-4999 (2008) [doi:10.1364/OE.16.004991].

[66] K. Sasaki, J. Hotta, K. Wada, and H. Masuhara, "Analysis of radiation pressure exerted on a metallic particle within an evanescent field," Opt. Lett. 25(18), 1385-1387 (2000) [doi:10.1364/OL.25.001385].

[67] J. Arias-Gonzalez and M. Nieto-Vesperinas, "Optical forces on small particles: attractive and repulsive nature and plasmon-resonance conditions," J. Opt. Soc. Am. A 20(7), 12011209 (2003) [doi:10.1364/JOSAA.20.001201].

[68] A. Zelenina, R. Quidant, G. Badenes, and M. Nieto-Vesperinas, “Tunable optical sorting and manipulation of nanoparticles via plasmon excitation," Opt. Lett. 31(13), 2054-2056 (2006) [doi:10.1364/OL.31.002054].

[69] M. Pelton, M. Liu, H. Y. Kim, G. Smith, P. Guyot-Sionnest, and N. F. Scherer, "Optical trapping and alignment of single gold nanorods by using plasmon resonances," Opt. Lett. 31(13), 2075-2077 (2006) [doi:10.1364/OL.31.002075].

[70] K. Toussaint, M. Liu, M. , J. Pesic, M. Guffey, P. Guyot-Sionnest, and N. Scherer, "Plasmon resonance-based optical trapping of single and multiple Au nanoparticles," Opt. Express 15(19), 12017-12029 (2007) [doi:10.1364/OE.15.012017].

[71] C. Sönnichsen, Plasmons in Metal Nanostructures, Ludwig-Maximilians-Universität München (2001).

[72] T. Perkins, S. Quake, D. Smith, and S. Chu, "Relaxation of a single DNA molecule observed by optical microscopy," Science 264(5160), 822-826 (1994) [doi:10.1126/science.8171336].

[73] C. Asbury, A. Fehr, and S. Block, "Kinesin moves by an asymmetric hand-over-hand mechanism," Science 302(5653), 2130-2134 (2003) [doi:10.1126/science.1092985].

[74] C. Bustamante, Z. Bryant, and S. Smith, "Ten years of tension: single-molecule DNA mechanics," Nature 421, 423-427 (2003) [doi:10.1038/nature01405].

[75] D. Chiu and R. Zare, "Biased diffusion, optical trapping, and manipulation of single molecules in solution," J. Am. Chem. Soc 118(27), 6512-6513 (1996) [doi:10.1021/ja960978p].

[76] S. Katsura, K. Hirano, Y. Matsuzawa, K. Yoshikawa, A. Mizuno, and O. Journals, "Direct laser trapping of single DNA molecules in the globular state," Nucl. Acids Res. 26(21), 4943-4945 (1998) [doi:10.1093/nar/26.21.4943].

[77] M. Ichikawa, Y. Matsuzawa, and K. Yoshikawa, "Entrapping polymer chain in light well under good solvent condition," J. Phys. Soc. Jpn. 74(7), 1958-1961 (2005) [doi:10.1143/JPSJ.74.1958].

[78] H. Li, D. Zhou, H. Browne, and D. Klenerman, "Evidence for resonance optical trapping of individual fluorophore-labeled antibodies using single molecule fluorescence spectroscopy," J. Am. Chem. Soc 128(17), 5711-5717 (2006) [doi:10.1021/ja056997t].

[79] M. Osborne, S. Balasubramanian, W. Furey, and D. Klenerman, "Optically biased diffusion of single molecules studied by confocal fluorescence microscopy," J. Phys. Chem. B 102(17), 3160 (1998) [doi:10.1021/jp9715078].

[80] G. Chirico, C. Fumagalli, and G. Baldini, "Trapped brownian motion in single-and twoPhoton excitation fluorescence correlation experiments," J. Phys. Chem. B 106(10), 25082519 (2002) [doi:10.1021/jp013087z].

[81] W. Singer, T. Nieminen, N. Heckenberg, and H. Rubinsztein-Dunlop, "Collecting single molecules with conventional optical tweezers," Phys. Rev. E 75, 011916 (2007) [doi:10.1103/PhysRevE.75.011916]. 
[82] W. Lee, P. Reece, R. Marchington, N. Metzger, and K. Dholakia, "Construction and calibration of an optical trap on a fluorescence optical microscope," Nat. Protoc. 2(12), 32263238 (2007) [doi:10.1038/nprot.2007.446].

[83] K. Wulff, D. Cole, R. Clark, R. DiLeonardo, J. Leach, J. Cooper, G. Gibson, and M. Padgett, "Aberration correction in holographic optical tweezers," Opt. Express 14, 4169-4174 (2006) [doi:10.1364/OE.14.004169].

[84] E. Theofanidou, L. Wilson, W. Hossack, and J. Arlt, "Spherical aberration correction for optical tweezers," Opt. Commun. 236, 145-150 (2004) [doi:10.1016/j.optcom.2004.03.009].

[85] K. Neuman, E. Chadd, G. Liou, K. Bergman, and S. Block, "Characterization of Photodamage to Escherichia coli in Optical Traps," Biophys. J. 77(5), 2856-2863 (1999).

[86] A. Siegman, "Defining, measuring, and optimizing laser beam quality," Proceedings of SPIE 2(1993) (1993) [doi:10.1117/12.150601].

[87] B. Agate, C. Brown, W. Sibbett, and K. Dholakia, "Femtosecond optical tweezers for in-situ control of two-photon fluorescence," Opt. Express 12(13), 3011-3017 (2004) [doi:10.1364/OPEX.12.003011].

[88] P. Li, K. Shi, and Z. Liu, "Manipulation and spectroscopy of a single particle by use of white-light optical tweezers," Opt. Lett. 30, 156-158 (2005) [doi:10.1364/OL.30.000156].

[89] D. Murphy, Fundamentals of Light Microscopy and Electronic Imaging, Wiley-Liss New York (2001).

[90] http://www.microscopyu.com/index.html.

[91] http://www.olympusmicro.com/.

[92] T. Squires and S. Quake, "Microfluidics: Fluid physics at the nanoliter scale," Rev. Mod. Phys. 77(3), 977-1026 (2005) [doi:10.1103/RevModPhys.77.977].

[93] M. Allersma, F. Gittes, M. deCastro, R. Stewart, and C. Schmidt, “Two-Dimensional Tracking of ncd Motility by Back Focal Plane Interferometry," Biophys. J. 74(2), 10741085 (1998).

[94] K. Berg-Sorensen and H. Flyvbjerg, "Power spectrum analysis for optical tweezers," Rev. Sci. Instr. 75, 594-612 (2004) [doi:10.1063/1.1645654].

[95] G. Volpe, G. Kozyreff, and D. Petrov, "Backscattering position detection for photonic force microscopy,” J. Appl. Phys. 102, 084701 (2007) [doi:10.1063/1.2799047].

[96] J. Huisstede, K. van der Werf, M. Bennink, and V. Subramaniam, "Force detection in optical tweezers using backscattered light," Opt. Express 13, 1113-1123 (2005) [doi:10.1364/OPEX.13.001113].

[97] S. Keen, J. Leach, G. Gibson, and M. J. Padgett, "Comparison of a high-speed camera and a quadrant detector for measuring displacements in optical tweezers," J. Opt. Soc. Am. A 9, S264-S266 (2007).

Maria Dienerowitz is a PhD student at the School of Physics and Astronomy at the University of St Andrews. She is investigating plasmonic excitations in metal nanoparticles and their optical trapping properties. Her research interests include plasmonics, optical micromanipulation and holographic beamshaping. Maria holds a stipend from the "Stiftung der Deutschen Wirtschaft" for her postgraduate studies and an SPIE Scholarship.

Michael Mazilu is a post doctoral research fellow at the School of Physics and Astronomy, University of St. Andrews, Scotland. His current research interests are the modelling and understanding of optical forces, optical vortex light-matter interaction, Raman spectroscopy and its application in biophotonics. His earlier research has been on ultra-short pulse phase/chirp measurement and on modelling and characterisation of photonic crystals, multiple quantum wells and quantum dots for use in ultra-fast opto-electronic devices. 
Kishan Dholakia is Professor of Physics at the University of St Andrews, Scotland and an honorary adjunct Professor at the Center for Optical Sciences at the University of Arizona. He heads a large group working in various aspects of photonics including micromanipulation and biophotonics. He has published over 300 journal/conference papers and his group won the European Optics Prize in 2003. He was elected to the position of Fellow of the Royal Society of Edinburgh in 2007 and Fellow of the Optical Society of America in 2008. 